\title{
Kompetenzdiagnose im Wirtschaftsbereich Fußballsport: Das Kompetenzinventar Profifußball (KIPF)
}

\author{
Julian Decius ${ }^{1}$ \\ Angenommen: 26. März 2021 / Online publiziert: 21. April 2021 \\ (c) Der/die Autor(en) 2021
}

\section{Zusammenfassung}

In einer zunehmend professionalisierten Welt des Fußballsports wächst auch die Bedeutung einer theoretisch fundierten Kompetenzentwicklung bei Profifußballspielern. Mit dem Kompetenzinventar Profifußball (KIPF) wird in diesem Beitrag in der Zeitschrift Gruppe. Interaktion. Organisation. (GIO) ein Fragebogeninstrument vorgestellt, um die beruflichen Handlungskompetenzen im Berufsfeld Profifußball messbar zu machen. Das KIPF basiert auf einem Kompetenzstrukturmodell, dessen Entwicklung anhand einer Literaturrecherche und auf Grundlage von Experteninterviews dargestellt wird. Der Entwicklungsprozess der Fragebogenitems, die Durchführung einer explorativen sowie einer konfirmatorischen Faktorenanalyse und die Überprüfung psychometrischer Gütekriterien werden ausführlich beschrieben. Die Entwicklung und Validierung basieren auf den quantitativen Daten von insgesamt 427 Fußballspielern. Die finale Version des KIPF umfasst die acht Kompetenzdimensionen Taktisches Wissen, Wissen zu Ernährung und Gesundheit, Einsatz von Resilienzmethoden, Umgang mit Medien, Kommunikationsfähigkeit, Teamunterstützende Anpassungsfähigkeit, Mentale Stärke sowie Kritikfähigkeit. Jede Kompetenzdimension wird mit drei Items erfasst. Abschließend werden die Einsatzmöglichkeiten in der Praxis (für Trainer, Verbandsfunktionäre, Spielerberater, Profifußballer) sowie in der Wissenschaft vorgestellt und Möglichkeiten der Übertragung beispielsweise auf den Frauenfußball, andere kulturelle Kontexte und weitere Mannschaftssportarten diskutiert.

Schlüsselwörter Kompetenzen · Handlungskompetenzen · Kompetenzinventar · Kompetenzstrukturmodell · Profifußball · Fußballspieler · Leistung · Performanz

Dr. Julian Decius

julian.decius@uni-paderborn.de

1 Institut für Psychologie, Universität Paderborn, Warburger

Straße 100, 33098 Paderborn, Deutschland 


\title{
Competence diagnosis in football (soccer): The competence inventory professional football (KIPF)
}

\begin{abstract}
In an increasingly professionalised world of football, the importance of theoretically based competence development among professional football players is growing. With the Competence Inventory Professional Football (KIPF), this article in the journal Gruppe. Interaktion. Organisation. (GIO) presents a measure to operationalise professional competences of football players. The KIPF is based on a competence structure model which has been developed following the results of a literature research and expert interviews. The development process of the questionnaire items, the conduction of an explorative factor analysis as well as a confirmatory factor analysis, and the examination of psychometric quality criteria are described in detail. The KIPF development and validation are based on quantitative data of 427 football players. The final version of the KIPF comprises the following eight competence dimensions: Tactical Knowledge, Knowledge of Nutrition and Health, Use of Resilience Methods, Dealing with Media, Communication Skills, Team Supporting Adaptability, Mental Strength, and Criticism Capacity. Each competence dimension is covered by three items. Possible KIPF applications in practice (for coaches, officials, player agents, professional footballers) as well as in science are presented. Furthermore, issues of transfer to women's football, other cultural contexts and other team sports are discussed. The items (not yet validated) in English are available in the Electronic Supplementary Material (ESM) to this article.
\end{abstract}

Keywords Skills · Capabilities · Competence Inventory · Competence Structure Model · Professional Football (Soccer) · Football Player · Performance

\section{Einleitung}

Der Profifußball gilt als ein ungewöhnliches Berufsfeld: Die Konkurrenz um die „Arbeitsplätze“ als Spieler auf dem Feld ist extrem hoch, ebenso der Leistungsdruck (Haugaasen und Jordet 2012). Die mediale Öffentlichkeit verfolgt jede Regung der Spieler während der Berufsausübung, aber zunehmend auch darüber hinaus im Privatleben, sodass Profifußballspieler als Personen des öffentlichen Lebens angesehen werden können. Zudem hat sich die Branche in den letzten Jahrzehnten zu einem Millionengeschäft mit exorbitanten Gehältern, Prämienzahlungen, Ablösesummen und Handgeldern für Spielerwechsel sowie Beraterhonoraren entwickelt. Die Profifußballvereine - inzwischen fast ausschließlich in der Rechtsform von Kapitalgesellschaften (Fahrner 2014) - versuchen, die Voraussetzungen für den sportlichen Erfolg der Mannschaft durch immer ausgeprägtere Professionalisierung aller Bereiche zu verbessern. Im Fokus steht dabei die Performanz der Spieler. Performanz kann betrachtet werden als das Ergebnis einer Arbeitstätigkeit, im Sinne von Leistung (Apitzsch 2012; vgl. Max 1999). Bei der Förderung der Performanz - im Sport allgemein und im Fußballsport im Speziellen - kann u. a. zwischen den physischen bzw. physiologischen und technischen Komponenten einerseits und den psychologischen Komponenten andererseits unterschieden werden ${ }^{1}$ (vgl. Forsman et al. 2016a; Haugaasen und Jordet 2012). Während die Leistungsdiagnostik im physischen Bereich bereits als durchaus aus-

\footnotetext{
${ }^{1}$ Außerdem stellen „Glück“ und „Pech“ im Karriereverlauf der Fußballspieler relevante Faktoren für den langfristigen Erfolg im Profifußball dar (Decius 2018).
}

gereift bezeichnet werden kann (für einen Überblick siehe z. B. Ali 2011), finden psychologische Komponenten erst in den letzten beiden Jahrzehnten verstärkt in Forschung und Praxis des Profifußballs Beachtung - jedoch fast ausschließlich im Jugendbereich, nicht im Erwachsenenfußball (vgl. Feichtinger und Höner 2015; Gledhill et al. 2017; Gould et al. 2002; Holt und Dunn 2004; Höner und Feichtinger 2016; Murr et al. 2018b; Nesti und Sulley 2015; Van Yperen 2009).

Doch welche psychologischen Faktoren sind relevant für die Performanz im Profifußball? Als Vorbedingung der manifesten Performanz im Fußball (z. B. beobachtbar anhand von Wettkampfergebnissen und Leistungskennwerten) kann auf psychologischer Ebene das latente, d.h. nicht direkt beobachtbare Konstrukt der Kompetenz angesehen werden (Apitzsch 2012; vgl. Franke 2005). Gemäß Weinert (2001) kann Kompetenz definiert werden als

die bei Individuen verfügbaren oder durch sie erlernbaren kognitiven Fähigkeiten und Fertigkeiten, um bestimmte Probleme zu lösen, sowie die damit verbundenen motivationalen, volitionalen und sozialen Bereitschaften und Fähigkeiten, um die Problemlösungen in variablen Situationen erfolgreich und verantwortungsvoll nutzen zu können. (S. 27-28)

Bisherige Studien befassten sich vor allem mit der Kompetenzanalyse bei Trainern und Sportmanagern (Apitzsch 2012; Kaß 2013). Für die Zielgruppe der Athleten entwickelten Macnamara und Collins (2011) ein Instrument zur Messung psychologischer Charakteristiken für die Entwicklung sportlicher Exzellenz, welches teilweise kompetenzähnliche Items aufweist, jedoch auch Rahmenbedingungen 
wie Unterstützung durch Trainer beinhaltet. Außerdem bezogen sie sich dabei nicht spezifisch auf den Fußballsport, sondern Mannschaftssportarten im Allgemeinen, und fokussierten den Jugendbereich. Die „Self-perceived Soccer Competence Scale" von Ambe et al. (2013) enthält dagegen fast ausschließlich physische bzw. technische Aspekte wie Schnelligkeit, Ausdauer oder Kopfballstärke. Eine weitere, wiederum für Nachwuchsspieler konzipierte Skala, in der ebenfalls größtenteils physische bzw. technische Merkmale im Selbstbericht erfragt werden (z.B. Schnelligkeit und Beweglichkeit), stellten Forsman et al. (2016b) vor.

Die Bedeutung der Talententwicklung von Jugendspielern und die damit einhergehenden Studien sind zweifelsohne sehr wichtig - allerdings ist nicht davon auszugehen, dass ein Fußballspieler mit dem Eintritt in die Profimannschaft bezüglich seiner Kompetenzen vollständig entwickelt ist. Im Gegensatz zum Profifußball ist in anderen Berufsbereichen das Konzept des lebenslangen Lernens weit verbreitet (Kyndt et al. 2011; Livingstone 2008; Rainbird 2000). Zwar können Fußballspieler im besten Fall lediglich etwa 20 Jahre lang ihren Beruf ausüben, sodass der Terminus „lebenslanges Lernen“ übertrieben erscheint - ausgeprägtes Lernen während des Fußballberufes findet jedoch schon seit jeher statt, beispielsweise informell durch eigenes Ausprobieren, Modelllernen, Feedback und Reflektion (vgl. Decius et al. 2019). Allerdings sollte dieser Lernfokus nicht wie bisher nahezu ausschließlich auf der stetigen Optimierung der physischen, physiologischen und technischen Fähigkeiten und Fertigkeiten liegen, sondern auch psychologische Aspekte einbeziehen.

Um die (psychologischen) Kompetenzen der Profifußballspieler weiterentwickeln zu können, ist jedoch zunächst zu ermitteln, welche Kompetenzen für die Performanz im Profifußballsport bedeutsam sind. Dabei ist davon auszugehen, dass im Erwachsenenbereich auch Aspekte eine Rolle spielen, die im Jugendbereich noch als weniger wichtig eingestuft werden können. Hier wäre z.B. der professionelle Umgang mit der Presse und anderen Medien zu nennen wenngleich insbesondere die sozialen Medien, die auch von jungen Spielern verstärkt genutzt werden, für Spitzensportler zum Zweck der Selbstvermarktung eine immer größere Rolle spielen (Haupt und Schöttl 2016). Die Ermittlung der für erwachsene Profifußballer relevanten Kompetenzen bildet die Grundlage, um diese Kompetenzen anschließend operationalisieren zu können.

Im vorliegenden Beitrag wird daher auf Basis der Literatur und der Ergebnisse aus qualitativen Interviews mit Fußballspielern und Experten ein Kompetenzstrukturmodell - in dem die Dimensionalität der Kompetenzen dargestellt werden kann (Hartig und Klieme 2006) - für den Profifußball im Erwachsenenbereich aufgestellt. Auf Grundlage dieses Modells wird die Entwicklung und Validierung eines Instrumentariums zur Operationalisierung der für Profifußballspieler relevanten Kompetenzen vorgestellt: das Kompetenzinventar Profifußball (KIPF).

\section{Konzeptioneller Hintergrund: Entwicklung eines Kompetenzstrukturmodells}

Zur Dimensionierung des zu erstellenden Kompetenzstrukturmodells wurde die Taxonomie von Kauffeld (2006) verwendet. Kauffeld unterteilt berufliche Handlungskompetenz in folgende vier Bereiche: Fach-, Methoden-, Sozial- und Selbstkompetenz. Nach Kauffeld und Grote (2019) umfasst Fachkompetenz alle Kenntnisse, Fertigkeiten und Fähigkeiten, die sich auf die Organisation, Aufgaben, Prozesse sowie den eigenen Arbeitsplatz beziehen. Methodenkompetenz beinhaltet Techniken, Methoden und Vorgehensweisen zur Strukturierung der Arbeitsaufgaben. Sozialkompetenz bezieht sich auf die Fähigkeit, sich im sozialen Umgang situationsspezifisch angemessen zu verhalten, z.B. in Bezug auf Kommunikation und Kooperation. Bei der Selbstkompetenz geht es darum, wie Individuen innerhalb der Arbeit mit sich selbst umgehen, wozu beispielsweise die Bereitschaft zur Selbstreflexion und Leistungsbereitschaft zählen.

Zur deduktiven Ableitung von psychologischen Performanzbedingungen wurde eine Literaturrecherche durchgeführt. Dabei konnten 25 performanzförderliche Aspekte aus der bisherigen Forschung abgeleitet und in die Taxonomie der Handlungskompetenzen eingeordnet werden:

- im Bereich Fachkompetenz: Spieltechniken (Larsen et al. 2013; Mills et al. 2012), Wissen/Fertigkeiten (Larsen et al. 2013; Sygusch et al. 2014), Mentale Fähigkeiten zur Anwendung physischer Fertigkeiten (Mills et al. 2012)

- im Bereich Methodenkompetenz: Zielorientiertheit des Handelns/Zielsetzung (Gould et al. 2002; Larsen et al. 2013), Fokussierung (Macnamara und Collins 2011; Mills et al. 2012)

- im Bereich Sozialkompetenz: Kommunikationsfähigkeit (Macnamara und Collins 2011), Respekt und Freundlichkeit zeigen (Larsen et al. 2013), Teamfähigkeit (Gould et al. 2002; Larsen et al. 2013), Empathie (Mills et al. 2012), Soziale Anpassungsfähigkeit (Mills et al. 2012), Akzeptanz fremder Entscheidungen (Holt und Dunn 2004)

- im Bereich Selbstkompetenz: Selbstständigkeit (Cook et al. 2014), Reflexivität (Mills et al. 2012), Belastbarkeit (Cook et al. 2014; Gould et al. 2002; Holt und Dunn 2004), Engagement und unbeirrte Karriereplanung (Holt und Dunn 2004), Disziplin (Gledhill et al. 2017; Holt und Dunn 2004; Mills et al. 2012), Wille und Motivation (Gledhill et al. 2017; Holt und Dunn 2004; Larsen 
et al. 2013), Emotionale Stabilität (Gledhill et al. 2017), Selbstbewusstsein (Gould et al. 2002; Larsen et al. 2013; Mills et al. 2012), Umgang mit Niederlagen und Rückschlägen (Gould et al. 2002; Mills et al. 2012), Umgang mit Stress/Leistung unter Druck (Holt und Dunn 2004; Macnamara und Collins 2011; Mills et al. 2012), Positive Einstellung/Mentalität (Holt und Dunn 2004; Mills et al. 2012), Realistische Selbsteinschätzung (Gledhill et al. 2017; Gould et al. 2002; Macnamara und Collins 2011), Verantwortungsbewusstsein (Holt und Dunn 2004), Kritikfähigkeit (Cook et al. 2014; Mills et al. 2012)

Um das vorläufige Kompetenzstrukturmodell zu validieren, wurden 16 leitfadengestützte Interviews durchgeführt. Dazu wurde ein Interviewleitfaden entwickelt, um mittels der Critical-Incident-Technik (Flanagan 1954) herauszufinden, welche der Faktoren in für den Erfolg besonders förderlichen und hinderlichen Situationen eine wichtige Rolle einnehmen. Der Interviewleitfaden umfasste die drei Themenblöcke Spielereigenschaften, Training und Umgebung, sowie Mentale Aspekte. Im Vorfeld der Interviews wurde ein Pre-Test mit einem Experten aus dem Spielergewerkschaftsbereich durchgeführt und der Leitfaden überarbeitet. Befragt wurden anschließend zehn aktive männliche Fußballspieler (drei Spieler mit Spielerfahrung in der 1. Bundesliga, vier in der 2. Bundesliga, einer in der 3. Liga, zwei in der Regionalliga) sowie sechs im Fußballbereich tätige ExpertInnen $(5 \mathrm{~m}, 1 \mathrm{w})$ aus insgesamt drei unterschiedlichen Vereinen aus der 1. Bundesliga, 2. Bundesliga und 3. Liga (zwei SportpsychologInnen, zwei Mitarbeiter der Nachwuchsleitung und -betreuung, zwei Personen aus dem Managementbereich). Eine inhaltsanalytische Auswertung (Mayring 2010) ergab, wie oft die performanzförderlichen Aspekte in den Interviews genannt wurden. Zudem wurde das aus den 25 Aspekten bestehende Kategoriensystem auf Basis der Interviewdaten induktiv erweitert; Mayring (2010) bezeichnet das induktive Vorgehen bei der qualitativen Inhaltsanalyse als ,sehr fruchtbar“ und schreibt ihm eine große Bedeutung innerhalb qualitativer Ansätze zu (S. 85). Hinzugefügt wurden die folgenden sieben Aspekte (vgl. Anhang ${ }^{2}$ ): Umgang mit Medien (Methodenkompetenz); Hilfsbereitschaft, Wahrnehmung einer Vorbildfunktion (jeweils Sozialkompetenz); Durchhaltevermögen, Lernwilligkeit, Geduld, Bodenständigkeit (jeweils Selbstkompetenz).

In einem zweiten qualitativen Analyseschritt wurde die 16 Interviewdatensets daraufhin untersucht, inwieweit die

\footnotetext{
2 Tab. 5 im Anhang zeigt, wie oft die nun insgesamt 32 Kompetenzfacetten des erweiterten, jedoch noch nicht finalen Kompetenzstrukturmodells von den Spielern, von den ExpertInnen und von beiden Gruppen in Summe genannt wurden.
}

folgenden drei Kriterien bei den 32 Kompetenzfacetten zutreffen:

- Ausführlichkeit der Beschreibung (d.h., wie ausführlich und detailreich beschrieben die Interviewten den Inhalt der erwähnten Kompetenzaspekte in den Critical-Incident-Situationen)

- Zugeschriebene Wichtigkeit (d.h., welche Wichtigkeit schrieben die Interviewten den Kompetenzaspekten [implizit] für die Performanz und den Erfolg von Profifußballspielern $\mathrm{zu}$ )

- Sicherheit der eigenen Expertise (d.h., wie sicher und überzeugt waren die Interviewten bezüglich der Bewertung der Wichtigkeit des jeweils genannten Kompetenzaspektes)

Jedes Kriterium wurde mit 0, 1, 2 oder 3 bewertet, sodass sich bei drei Bewertungsausprägungen mal drei Kriterien mal 16 Interviews ein maximaler Relevanzwert von 144 ergeben konnte. Die Annahme war, dass eine Kompetenzfacette umso relevanter für die Performanz im Profifußball ist, je ausführlicher sie in den Critical-Incident-Situationen beschrieben wurde, je höher die ihr zugeschriebene Wichtigkeit war, und je sicherer sich die Interviewten bei der Einschätzung waren. Der Relevanzwert der Kompetenzfacetten ist ebenfalls in Tab. 5 (im Anhang) zu finden.

Zusätzlich wurde während des zweiten Analyseschritts untersucht, inwiefern die Kompetenzfacetten inhaltlich trennscharf oder aber inhaltlich sehr ähnlich zueinander sind. Drei in der Wissenschaft tätige Personen mit Expertise im Bereich Kompetenzentwicklung bzw. Fußballsport diskutierten daraufhin die vermeintlich ähnlichen Kompetenzfacetten. Auf Basis dieser Fachdiskussion wurden ähnliche Kompetenzfacetten zusammengelegt, sodass sich im finalen Kompetenzstrukturmodell 16 Kompetenzen befinden (siehe Tab. 1), von denen zwei der Fachkompetenz, drei der Methodenkompetenz, drei der Sozialkompetenz und acht der Selbstkompetenz zuzuordnen sind. Außerdem wurde die Facette ,Mentale Fähigkeiten zur Anwendung physischer Fertigkeiten“ in den griffigeren Terminus „Wissen zu Gesundheit und Lebensführung“ umbenannt, da die kodierten Interviewstellen fast ausschließlich Inhalte zur Umsetzung eines gesunden Lebensstils, Ernährung oder Regeneration enthielten.

\section{Entwicklungsschritte zur Erstellung des KIPF}

\subsection{Entwicklung des Itempools}

Für die Entwicklung des Kompetenzinventars Profifußball (KIPF) wurde basierend auf dem finalen Kompetenzstrukturmodell und den Nennungen von Aussagen und Themen 
Tab. 1 Finales Kompetenzstrukturmodell für den Profifußball

\begin{tabular}{|c|c|}
\hline \multirow[t]{2}{*}{ Fachkompetenz } & $\begin{array}{l}\text { Wissen zu Gesundheit und Lebensführung (vorher benannt als: Mentale Fähigkeiten zur Anwendung physischer Fer- } \\
\text { tigkeiten) }\end{array}$ \\
\hline & Taktisches Wissen (neu zusammengefügt aus: Wissen/Fertigkeiten; Spieltechniken) \\
\hline \multirow[t]{3}{*}{ Methodenkompetenz } & Zielorientiertheit des Handelns/Zielsetzung \\
\hline & Umgang mit Medien \\
\hline & $\begin{array}{l}\text { Methoden zur Fokussierung und zum Umgang mit Stress (neu zusammengefügt aus: Fokussierung; Umgang mit } \\
\text { Stress/Leistung unter Druck) }\end{array}$ \\
\hline \multirow[t]{3}{*}{ Sozialkompetenz } & Kommunikationsfähigkeit \\
\hline & $\begin{array}{l}\text { Teamunterstützung (neu zusammengefügt aus: Respekt und Freundlichkeit zeigen; Teamfähigkeit; Wahrnehmung einer } \\
\text { Vorbildfunktion; Hilfsbereitschaft; Empathie) }\end{array}$ \\
\hline & $\begin{array}{l}\text { Soziale Anpassungsfähigkeit und Akzeptanz (nеu zusammengelegt aus: Soziale Anpassungsfähigkeit; Akzeptanz frem- } \\
\text { der Entscheidungen) }\end{array}$ \\
\hline \multirow[t]{8}{*}{ Selbstkompetenz } & Selbstbewusstsein \\
\hline & Disziplin \\
\hline & Kritikfähigkeit \\
\hline & $\begin{array}{l}\text { Mentale Stärke (neu zusammengefügt aus: Durchhaltevermögen; Belastbarkeit; Umgang mit Niederlagen und Rückschlä- } \\
\text { gen; Emotionale Stabilität) }\end{array}$ \\
\hline & Reflexionsfähigkeit (nеu zusammengefügt aus: Reflexivität; Realistische Selbsteinschätzung) \\
\hline & $\begin{array}{l}\text { Motivation und Engagement (neu zusammengefügt aus: Wille und Motivation; Engagement und unbeirrte Karrierepla- } \\
\text { nung; Lernwilligkeit; Positive Einstellung/Mentalität) }\end{array}$ \\
\hline & Bodenständigkeit und Geduld (neu zusammengefügt aus: Bodenständigkeit; Geduld) \\
\hline & $\begin{array}{l}\text { Verantwortungsbewusstsein und Selbständigkeit (nеu zusammengefügt aus: Verantwortungsbewusstsein; Selbstständig- } \\
\text { keit) }\end{array}$ \\
\hline
\end{tabular}

in den Interviews ein Itempool zusammengestellt. Dazu wurden insgesamt 157 Items entwickelt (gemäß Empfehlungen von DeVellis 2003 und MacKenzie et al. 2011), die inhaltlich alle 16 Kompetenzen des Kompetenzstrukturmodells abbildeten. Bei der Itemformulierung wurde darauf geachtet, dass die Items für die Zielgruppe der Fußballspieler verständlich und ohne großen kognitiven Aufwand erfassbar sind, und dass eine hohe Augenscheinvalidität gewährleistet ist, um die Akzeptanz der Items zu erhöhen (vgl. Döring und Bortz 2016). Daher wurde beispielsweise darauf verzichtet, einen schematischen Aufbau mit festen Itemstämmen zu verwenden. Anschließend wurden vier ausführliche Expertengespräche mit Personen durchgeführt, die im Profifußballbereich arbeiten. Ziel der Gespräche war es, unter den teils sehr ähnlichen Items zu manchen Themen die inhaltlich validesten auszuwählen. Außerdem sollten die Experten prüfen, ob die sprachliche Formulierung der Items für die Zielgruppe der Profi-Fußballspieler angemessen ist. Die Ergebnisse der Gespräche legten die Entfernung von 47 Items sowie die Umformulierung von einem Item nahe. Die Experten sahen hingegen keinen Ergänzungsbedarf in Hinblick auf möglicherweise zu inhaltlich zu schmal abgedeckte Kompetenzfacetten. Der überarbeitete Itempool umfasste somit 110 Items.

\subsection{Pilotierungsstudie}

Anschließend wurde eine Pilotierungsstudie durchgeführt. Ziel dieser Studie war es, den immer noch umfangreichen
Itempool unter Einbezug erster deskriptiv-statistischer Erkenntnisse weiter zu kürzen, um den Teilnehmern der Hauptstudie - die auch Validierungskonstrukte umfassen sollte - keinen unzumutbar langen Fragebogen vorlegen zu müssen. Für die Pilotierungsstudie wurden 54 männliche Fußballspieler (Alter in Kategorien: <18 Jahre: 1 Person; 18-20: 15; 21-23: 9; 24-26: 19; 27-29: 8; >35: 2) aus verschiedenen Vereinen akquiriert, die den 110 Kompetenzitems umfassenden Fragebogen als Online-Version (Unipark) bearbeiteten. Die Items wurden im Fragebogen mit einer sechsstufigen Likert-Skala erfasst: „Stimme überhaupt nicht zu“, „Stimme nicht zu“, „Stimme wenig zu“, „Stimme etwas zu“, „Stimme zu“ und „Stimme voll zu“.

Für die intendierte Straffung des Itempools wurden die Items von drei in der Wissenschaft tätigen Personen diskutiert und dabei - neben des Iteminhalts - die Itemschwierigkeit (eine mittlere Schwierigkeit wurde bevorzugt; vgl. Döring und Bortz 2016; Moosbrugger und Kelava 2012), die Standardabweichung (eine hohe Standardabweichung wurde bevorzugt) sowie Schiefe und Kurtosis (die möglichst nicht außerhalb des Bereichs von -2 bis +2 liegen sollten; Kline 2016, S. 76) als Hinweise auf die Normalverteilung der Items berücksichtigt. Unter Berücksichtigung dieser Kriterien wurden - sofern keine inhaltlichen Gründe dagegensprachen - der Itempool reduziert. Insgesamt wurden auf diese Weise 47 Items aus dem zuvor 110 Items umfassenden Itempool entfernt. Außerdem wurden vier Items umformuliert und sechs Items ergänzt. Bei den Umformulierungen wurde beispielsweise der Kontext des Items 
Tab. 2 Items und Faktorladungen der Explorativen Faktorenanalyse (Mustermatrix)

\begin{tabular}{|c|c|c|c|c|c|c|c|c|}
\hline Items & 1 & 2 & 3 & 4 & 5 & 6 & 7 & 8 \\
\hline $\begin{array}{l}\text { Wenn mich großer Stress von meinen Zielen ablenkt, } \\
\text { nutze ich bestimme Methoden, um mich wieder fokus- } \\
\text { sieren zu können }\end{array}$ & 0,89 & - & - & - & - & - & - & - \\
\hline $\begin{array}{l}\text { Ich nutze Entspannungstechniken immer dann, wenn } \\
\text { mir alles zu viel wird }\end{array}$ & 0,84 & - & - & - & - & - & - & - \\
\hline $\begin{array}{l}\text { Wenn ich gestresst oder nervös bin, wende ich Atemtech- } \\
\text { niken oder ähnliche Methoden an, um mich zu entspan- } \\
\text { nen }\end{array}$ & 0,77 & - & - & - & - & - & - & - \\
\hline $\begin{array}{l}\text { Gewisse Techniken helfen mir, meine Ziele nach Wich- } \\
\text { tigkeit zu ordnen }\end{array}$ & 0,77 & - & - & - & - & - & - & - \\
\hline $\begin{array}{l}\text { Ich bin ein großer Fan von Übungen, die mir den Stress } \\
\text { nehmen }\end{array}$ & 0,72 & - & - & - & - & - & - & - \\
\hline Ich kenne mich mit Methoden zur Entspannung aus & 0,67 & - & - & - & - & - & - & - \\
\hline $\begin{array}{l}\text { Ich nutze bestimmte Methoden, um meine persönlichen } \\
\text { Trainingsziele zu erreichen }\end{array}$ & 0,62 & - & - & - & - & - & - & - \\
\hline $\begin{array}{l}\text { Ich stelle mir meine Ziele bildlich vor, um sie besser zu } \\
\text { erreichen }\end{array}$ & 0,43 & - & - & - & - & - & - & - \\
\hline Gesunde Ernährung ist für mich ein wichtiges Thema & - & 0,90 & - & - & - & - & - & - \\
\hline $\begin{array}{l}\text { Ich achte im hohen Maße darauf, mich gesund zu } \\
\text { ernähren }\end{array}$ & - & 0,89 & - & - & - & - & - & - \\
\hline $\begin{array}{l}\text { Mir ist bewusst, wie ich mich durch meine Ernäh- } \\
\text { rungsweise vor Verletzungen schützen kann }\end{array}$ & - & 0,74 & - & - & - & - & - & - \\
\hline $\begin{array}{l}\text { Über die Themen Ernährung, Schlaf und Gesundheit weiß } \\
\text { ich sehr gut Bescheid }\end{array}$ & - & 0,68 & - & - & - & - & - & - \\
\hline $\begin{array}{l}\text { Ich weiß im Detail, welche Auswirkungen meine Schlaf- } \\
\text { menge auf meine Leistung hat }\end{array}$ & - & 0,52 & - & - & - & - & - & - \\
\hline $\begin{array}{l}\text { Mein Fachwissen im Fußball ist so hoch, dass ich Trai- } \\
\text { ner werden könnte }\end{array}$ & - & - & 0,89 & - & - & - & - & - \\
\hline $\begin{array}{l}\text { Ich weiß sehr viel über taktische Feinheiten im Fuß- } \\
\text { ball }\end{array}$ & - & - & 0,77 & - & - & - & - & - \\
\hline $\begin{array}{l}\text { Ich habe ein sehr großes Verständnis für verschiedene } \\
\text { Fußballtaktiken }\end{array}$ & - & - & 0,71 & - & - & - & - & - \\
\hline $\begin{array}{l}\text { Durch mein hohes Wissen über den Fußball könnte ich als } \\
\text { Taktik-Experte im Fernsehen auftreten }\end{array}$ & - & - & 0,68 & - & - & - & - & - \\
\hline $\begin{array}{l}\text { Wenn wir neue Laufwege im Training einstudieren, kann } \\
\text { ich sie schneller verinnerlichen als meine Kollegen }\end{array}$ & - & - & 0,45 & - & - & - & - & - \\
\hline $\begin{array}{l}\text { Ich löse Probleme, indem ich direkt mit meinen Mit- } \\
\text { spielern spreche }\end{array}$ & - & - & - & 0,77 & - & - & - & - \\
\hline $\begin{array}{l}\text { Wenn ich ein Problem mit einem Mitspieler habe, } \\
\text { spreche ich ihn darauf an }\end{array}$ & - & - & - & 0,75 & - & - & - & - \\
\hline $\begin{array}{l}\text { Bei Schwierigkeiten mit Mitspielern hilft es mir, mit } \\
\text { ihnen zu reden }\end{array}$ & - & - & - & 0,66 & - & - & - & - \\
\hline Auf dem Platz bin ich ein kommunikativer Typ & - & - & - & 0,46 & - & - & - & - \\
\hline $\begin{array}{l}\text { Wenn ich merke, dass ein Spieler sportliche Probleme hat, } \\
\text { versuche ich ihm zu helfen }\end{array}$ & - & - & - & 0,42 & - & - & - & - \\
\hline Ich kläre oft Probleme zwischen meinen Mitspielern & - & - & - & 0,40 & - & - & - & - \\
\hline Ich fühle mich sicher im Umgang mit den Medien & - & - & - & - & 0,85 & - & - & - \\
\hline $\begin{array}{l}\text { Ich bin souverän im Umgang mit Journalisten und } \\
\text { Reportern }\end{array}$ & - & - & - & - & 0,73 & - & - & - \\
\hline Ich bin gut darin, öffentlich Interviews zu geben & - & - & - & - & 0,71 & - & - & - \\
\hline $\begin{array}{l}\text { Ich bin mir sehr bewusst, wie mein Verhalten die öffentli- } \\
\text { che Wahrnehmung von mir prägt }\end{array}$ & - & - & - & - & 0,70 & - & - & - \\
\hline $\begin{array}{l}\text { Ich weiß, wie ich mich in der Öffentlichkeit am besten } \\
\text { präsentieren kann }\end{array}$ & - & - & - & - & 0,51 & - & - & - \\
\hline $\begin{array}{l}\text { Ich zweifle an mir selbst, wenn ich im Spiel Fehler } \\
\text { mache. (invers) }\end{array}$ & - & - & - & - & - & 0,76 & - & - \\
\hline
\end{tabular}


Tab. 2 (Fortsetzung)

\begin{tabular}{|c|c|c|c|c|c|c|c|c|}
\hline Items & 1 & 2 & 3 & 4 & 5 & 6 & 7 & 8 \\
\hline $\begin{array}{l}\text { Ich schaffe es, auch unter enormen Druck, meine Leis- } \\
\text { tung im Spiel komplett abzurufen }\end{array}$ & - & - & - & - & - & 0,66 & - & - \\
\hline $\begin{array}{l}\text { Nervosität belastet mich während des Spiels sehr. (in- } \\
\text { vers) }\end{array}$ & - & - & - & - & - & 0,62 & - & - \\
\hline Ich bin ein sehr selbstbewusster Spieler & - & - & - & - & - & 0,50 & - & - \\
\hline $\begin{array}{l}\text { Wenn meine Mannschaft von den eigenen Fans ausgepfif- } \\
\text { fen wird, schaffe ich es trotzdem, mich voll und ganz auf } \\
\text { das Spiel zu konzentrieren }\end{array}$ & - & - & - & - & - & 0,47 & - & - \\
\hline $\begin{array}{l}\text { Wenn ich beim Elfmeterschießen als letzter ran soll, traue } \\
\text { ich mir das natürlich zu }\end{array}$ & - & - & - & - & - & - & - & - \\
\hline $\begin{array}{l}\text { Ich nutze jede Form der Kritik, um meine Spielweise } \\
\text { zu verbessern }\end{array}$ & - & - & - & - & - & - & 0,70 & - \\
\hline $\begin{array}{l}\text { Ich kann es gar nicht haben, wenn ich kritisiert werde. } \\
\text { (invers) }\end{array}$ & - & - & - & - & - & - & 0,68 & - \\
\hline $\begin{array}{l}\text { Jede Form der Kritik hilft mir, mich im Fußball zu } \\
\text { verbessern }\end{array}$ & - & - & - & - & - & - & 0,56 & - \\
\hline $\begin{array}{l}\text { Wenn ich von Reportern mehrfach das Gleiche gefragt } \\
\text { werde, werde ich ungemütlich. (invers) }\end{array}$ & - & - & - & - & - & - & 0,54 & - \\
\hline $\begin{array}{l}\text { Kritik an meiner Spielweise belastet mich emotional. } \\
\text { (invers) }\end{array}$ & - & - & - & - & - & 0,41 & 0,50 & - \\
\hline Wenn ich viel Geld verdiene, soll das jeder sehen. (invers) & - & - & - & - & - & - & 0,41 & - \\
\hline $\begin{array}{l}\text { Ich befolge auch Anweisungen des Trainers, die mir nicht } \\
\text { gefallen }\end{array}$ & - & - & - & - & - & - & 0,37 & - \\
\hline $\begin{array}{l}\text { Auch wenn ich mir unsicher bin, wechsle ich den Ver- } \\
\text { ein - Hauptsache das Geld stimmt. (invers) }\end{array}$ & - & - & - & - & - & - & 0,33 & - \\
\hline $\begin{array}{l}\text { Ich beschwere mich oft über die Entscheidung des Trai- } \\
\text { ners, wenn sie mir nicht passt. (invers) }\end{array}$ & - & - & - & - & - & - & 0,32 & - \\
\hline $\begin{array}{l}\text { Wenn wir ein Spiel verloren haben, suche ich die Fehler } \\
\text { immer zuerst bei mir selbst }\end{array}$ & - & - & - & - & - & - & - & - \\
\hline Ich respektiere immer die Entscheidung des Trainers & - & - & - & - & - & - & - & 0,73 \\
\hline $\begin{array}{l}\text { Wenn ich das Spiel von der Bank oder der Tribüne } \\
\text { verfolge, freue ich mich genauso über einen Erfolg } \\
\text { meiner Mannschaft, als wenn ich selbst spielen würde }\end{array}$ & - & - & - & - & - & - & - & 0,61 \\
\hline $\begin{array}{l}\text { Mein Erfolg ist mir egal - Hauptsache die Mannschaft } \\
\text { ist erfolgreich }\end{array}$ & - & - & - & 0,36 & - & - & - & 0,52 \\
\hline $\begin{array}{l}\text { Ich unterstütze meine Mannschaft auch dann, wenn ich } \\
\text { nicht spielen kann }\end{array}$ & - & - & - & - & - & - & - & 0,49 \\
\hline
\end{tabular}

Ergebnisse der Hauptachsenanalyse mit Promax-Rotation, Vorgabe: 8 Faktoren. Aus Gründen der Übersichtlichkeit sind niedrige Faktorladungen $(<0,3)$ nicht dargestellt. Fett gedruckte Items wurden für die finale Fragebogenversion ausgewählt

geändert (z.B. „Ich löse private Probleme, indem ich mit meinen Mitspielern spreche.“ $\rightarrow$ „Ich löse Probleme, indem ich direkt mit meinen Mitspielern spreche.“; Hinweis war hier eine hohe Itemschwierigkeit, d. h. eine niedrige Zustimmungstendenz zu dem Item, die vermutlich durch die Rahmung des privaten Kontextes entstand). Von den sechs neu hinzugefügten Items betrafen fünf den Bereich „Umgang mit Medien“, in dem bei der Reduktion zuvor viele nicht adäquate Items gestrichen worden waren. Im modifizierten Itempool verblieben nach diesen Anpassungen letztendlich 69 Items.

\subsection{Analysestrategie der Hauptstudie}

Im Anschluss daran wurde die Hauptstudie durchgeführt. Die Ziele der Hauptstudie waren, die am besten passenden Items für das Kompetenzinventar Profifußball (KIPF) auszuwählen, eine Faktorenstruktur im Itempool aufzudecken und diese Struktur als stabil zu bestätigen. Dazu wurde eine Stichprobe von 373 männlichen Fußballspielern akquiriert (Alter bekannt bei 133 Teilnehmern, $\mathrm{M}=21,1$; $\mathrm{SD}=6,0$ ), die sich aus fünf unabhängigen Akquise-Runden ergab. 2,4\% der Teilnehmer spielten in der 1. Bundesliga, $5,7 \%$ in der 2. Bundesliga, $14,1 \%$ in der 3 . Liga, $22,4 \%$ in den Regionalligen, $25,7 \%$ in den Oberligen, und 10,5\% in einer niedrigeren Liga, außerdem $19,2 \%$ in der U17- 


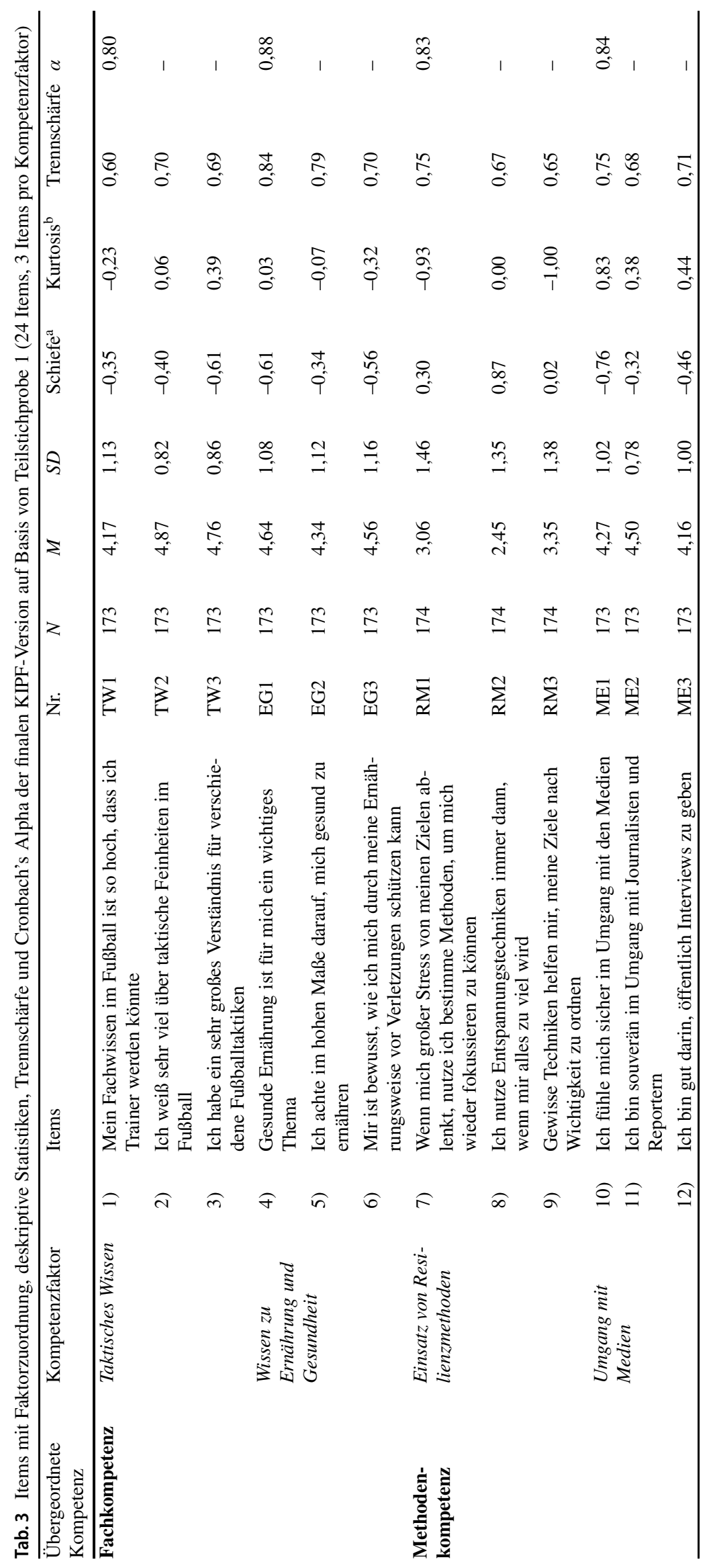




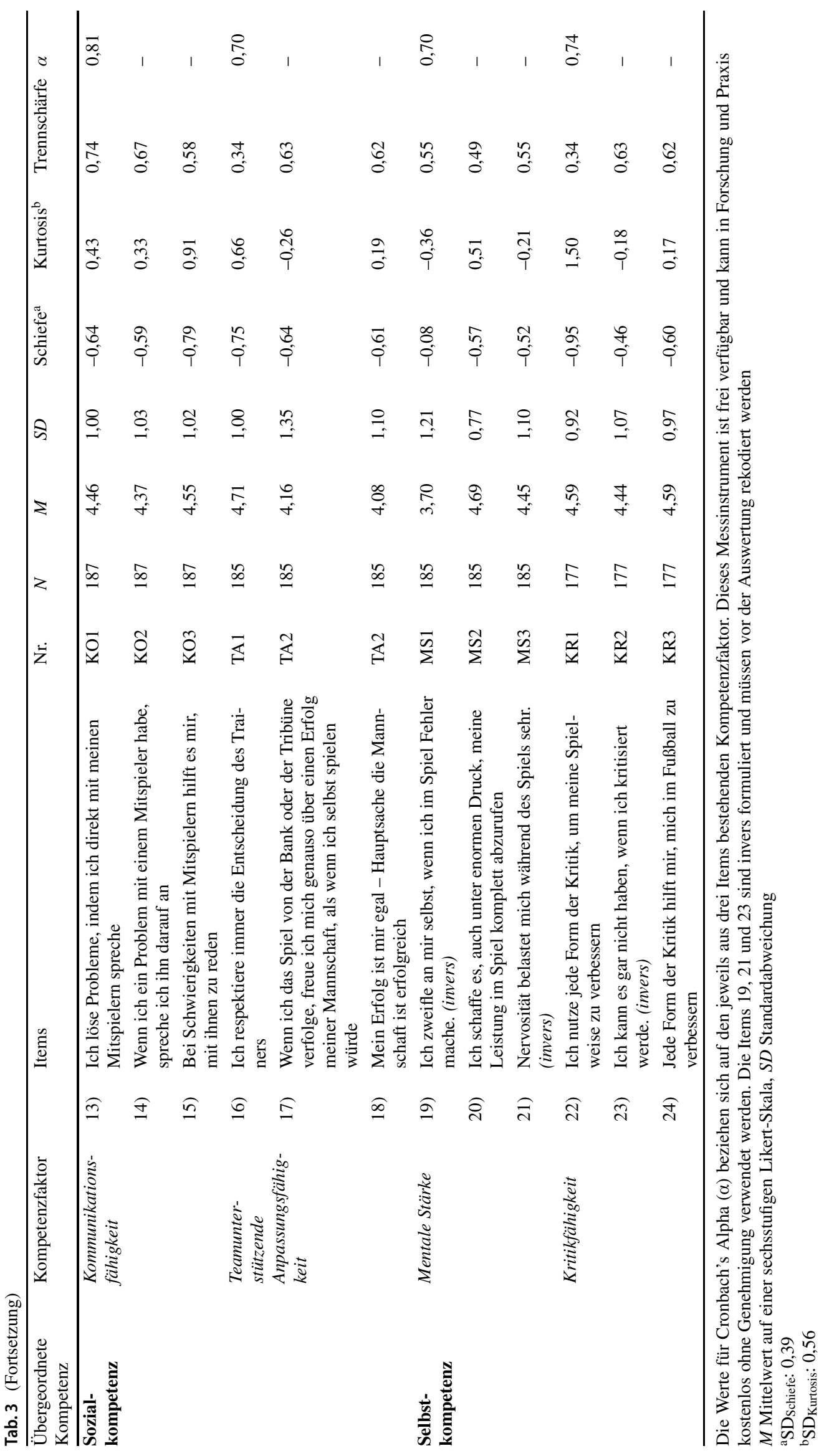


oder U19-Jugend-Bundesliga und damit in der höchstmöglichen Liga ihrer Altersklasse. Wie in der Pilotierungsstudie erfolgte die Datenerhebung auch in der Hauptstudie mit einem Online-Fragebogen (Unipark), der die 69 Items des modifizierten Itempools enthielt.

Als erster Schritt wurde der immer noch umfangreiche Itempool im Vorfeld der folgenden Analysen weiter reduziert. Dazu wurde erneut die Itemschwierigkeit betrachtet und nur Items im Itempool belassen, die eine mittlere Itemschwierigkeit zwischen 25 und 75 aufwiesen (und somit in den mittleren $50 \%$ der Itemschwierigkeit lagen). Von den 69 Items verblieben 49 im Itempool.

Um für die Durchführung der explorativen und der konfirmatorischen Faktorenanalyse zwei unabhängige Datensets $\mathrm{zu}$ erhalten, wurde der Gesamtdatensatz mittels $\mathrm{Zu}$ fallsalgorithmus in Excel in zwei Hälften geteilt. Die erste Hälfte $(N=187)$ wurde für die explorative Faktorenanalyse (EFA) verwendet, die zweite Hälfte $(N=186)$ für die konfirmatorische Faktorenanalyse (CFA). Die Berechnungen der EFA wurden mit der Statistiksoftware SPSS 25 durchgeführt; die CFA wurde mit der Statistiksoftware R (Version 3.6.1, R Core Team 2019) mit dem R-Paket lavaan (Rosseel 2012) berechnet.

\subsection{Explorative Faktorenanalyse}

Die Teilstichprobe zeigte sich als geeignet zur Durchführung einer EFA $(\mathrm{KMO}=0,77, \quad$ Bartlett-Test: $\chi^{2}(1776)=4460,91, p<0,00 ; \mathrm{vgl}$. Hutcheson und Sofroniou 1999). Die Stichprobengröße erwies sich als noch ausreichend für die Berechnung einer EFA, da $N>100$ ist und das Probanden-zu-Item-Verhältnis bei 3,8 und somit über dem empfohlenen Minimalwert von 3 liegt (MacKenzie et al. 2011, S. 310). Als Extraktionsmethode für die EFA wurde die Hauptachsenanalyse gewählt; zur besseren Interpretierbarkeit der Ergebnisse wurde mit der Promax-Rotation ein obliques Rotationsverfahren eingesetzt (Costello und Osborne 2005). Um die Faktorenanzahl zu bestimmen, wurde die Parallelanalyse verwendet (Horn 1965), die anderen Verfahren wie der Daumenregel ,Eigenwert $>1$ “ überlegen ist (Conway und Huffcutt 2003).

Die Parallelanalyse ergab eine anzunehmende Faktorenanzahl von acht. In Tab. 2 ist das Ergebnis der EFA (Mustermatrix) zu finden. Die acht Faktoren lassen sich inhaltlich interpretieren. Faktor 1 umfasst den Einsatz von Resilienzmethoden, Faktor 2 das Wissen zu Ernährung und Gesundheit, Faktor 3 das taktische Wissen, Faktor 4 die Kommunikationsfähigkeit, Faktor 5 den Umgang mit Medien, Faktor 6 die mentale Stärke, Faktor 7 die Kritikfähigkeit, und Faktor 8 die teamunterstützende Anpassungsfähigkeit.

Um das Ziel eines möglichst sparsamen und ökonomischen Messinstruments zu erreichen (Döring und Bortz 2016), wurden für jeden der acht Faktoren die drei Items mit der höchsten Faktorladung für das KIPF ausgewählt, sofern keine inhaltlichen Gründe dagegensprachen. Bei dem Faktor Einsatz von Resilienzmethoden wiesen zwei Items eine Faktorladung von 0,77 auf; um die inhaltliche Breite des Faktors zu gewährleisten und den Aspekt der Entspannungstechniken nicht doppelt abzudecken, wurde folgendes Item ausgewählt „,Gewisse Techniken helfen mir, meine Ziele nach Wichtigkeit zu ordnen.“(vgl. Tab. 2). Das Item „Mein Erfolg ist mir egal - Hauptsache die Mannschaft ist erfolgreich." aus dem Faktor Teamunterstützende Anpassungsfähigkeit wies eine Querladung von 0,36 auf den Faktor Kommunikationsfähigkeit auf, die aber als noch akzeptabel eingestuft wurde. Letztendlich ergab sich so eine 24 Items umfassende finale Version des KIPF. Drei Items sind invers formuliert und müssen vor der Auswertung der Skala rekodiert werden (vgl. Tab. 3). Die EFA-Ladungen dieser Items liegen zwischen 0,52 und 0,90 (vgl. Tab. 2); die Trennschärfe-Werte liegen zwischen 0,34 und 0,84 (vgl. Tab. 3), was als zufriedenstellend interpretiert werden kann (Lienert und Raatz 1998).

Zur Bestimmung der Reliabilität wurde Cronbach's Alpha als Maß für die interne Konsistenz der Skalen herangezogen. Die Alpha-Werte der ersten Teilstichprobe liegen zwischen 0,70 und 0,88 (vgl. Tab. 3) und damit in einem zufriedenstellenden Bereich (Hair et al. 2010). Bei Verwendung der Gesamtstichprobe ergeben sich Alpha-Werte zwischen 0,63 und 0,88 (vgl. Tab. 4) - hier weist das Konstrukt Mentale Stärke mit dem geringsten Alpha-Wert eine gerade noch akzeptable interne Konsistenz auf.

\subsection{Konfirmatorische Faktorenanalyse}

Anschließend wurde mit der zweiten Teilstichprobe eine konfirmatorische Faktorenanalyse (CFA) durchgeführt, um die zuvor explorativ abgeleitete Faktorenstruktur bestätigen zu können. Abb. 1 zeigt das Messmodell der CFA. Die acht Faktoren wurden als kovariierend modelliert (einen Überblick zu den Kovarianzen gibt Tab. 5 im Anhang). Zur Bewertung der Modellgüte wurde der globale Chi²-Wert sowie die folgenden, von Kline (2016, S. 269) empfohlenen, lokalen Modellgütekriterien herangezogen: Comparative Fit Index (CFI), Standardized Root Mean Square (SRMR), Root Mean Square Error of Approximation (RMSEA). Der CFI sollte über 0,95 liegen, der SRMR unter 0,08 und der RMSEA unter 0,06 (MacKenzie et al. 2011, S. 313). Die Ergebnisse der CFA zeigen eine akzeptable Modelgüte, $\chi^{2} / \mathrm{df}=295,637 / 224=1,32, \quad p=0,001 ; \quad \mathrm{CFI}=0,957$; $\mathrm{SRMR}=0,066 ; \mathrm{RMSEA}=0,041,90 \% \mathrm{CI}=[0,027,0,054]$. 
Tab. 4 Deskriptive Statistiken und Korrelationen auf Basis des Gesamtdatensatzes

\begin{tabular}{|c|c|c|c|c|c|c|c|c|c|c|c|c|c|c|}
\hline & Variable & $M$ & $S D$ & 1 & 2 & 3 & 4 & 5 & 6 & 7 & 8 & 9 & 10 & 11 \\
\hline 1 & Taktisches Wissen & 4,61 & 0,79 & 0,78 & - & - & - & - & - & - & - & - & - & - \\
\hline 2 & $\begin{array}{l}\text { Wissen zu Ernäh- } \\
\text { rung und Gesundheit }\end{array}$ & 4,44 & 1,03 & $0,25^{* *}$ & 0,85 & - & - & - & - & - & - & - & - & - \\
\hline 3 & $\begin{array}{l}\text { Einsatz von Resili- } \\
\text { enzmethoden }\end{array}$ & 2,99 & 1,19 & 0,04 & $0,37^{* *}$ & 0,83 & - & - & - & - & - & - & - & - \\
\hline 4 & Umgang mit Medien & 4,26 & 0,95 & $0,32^{* *}$ & $0,27^{* *}$ & $0,16^{* *}$ & 0,88 & - & - & - & - & - & - & - \\
\hline 5 & $\begin{array}{l}\text { Kommunikations- } \\
\text { fähigkeit }\end{array}$ & 4,53 & 0,82 & $0,14^{* *}$ & 0,10 & $0,13^{*}$ & $0,18^{* *}$ & 0,81 & - & - & - & - & - & - \\
\hline 6 & $\begin{array}{l}\text { Teamunterstützende } \\
\text { Anpassungsfähigkeit }\end{array}$ & 4,36 & 0,93 & 0,03 & $-0,06$ & $0,15^{* *}$ & $-0,07$ & $0,26^{* *}$ & 0,69 & - & - & - & - & - \\
\hline 7 & Mentale Stärke & 4,30 & 0,82 & $0,12^{*}$ & 0,10 & $-0,03$ & $0,19^{* *}$ & $0,27^{* *}$ & 0,05 & 0,57 & - & - & - & - \\
\hline 8 & Kritikfähigkeit & 4,46 & 0,84 & 0,03 & $0,14^{*}$ & $0,13^{*}$ & 0,06 & $0,23^{* *}$ & $0,25^{* *}$ & $0,19^{* *}$ & 0,72 & - & - & - \\
\hline 9 & $\begin{array}{l}\text { Fußballbezogene } \\
\text { Selbstwirksamkeit }\end{array}$ & 4,81 & 0,63 & $0,44^{* *}$ & $0,38^{* *}$ & $0,26^{* *}$ & $0,25^{* *}$ & $0,35^{* *}$ & 0,09 & $0,39^{* *}$ & $0,25^{* *}$ & 0,80 & - & - \\
\hline 10 & $\begin{array}{l}\text { Hoffnung auf Erfolg } \\
\text { (Sport) }\end{array}$ & 4,88 & 0,67 & $0,32^{* *}$ & $0,27^{* *}$ & $0,21^{* *}$ & 0,09 & $0,16^{*}$ & 0,10 & $0,21^{* *}$ & $0,23^{* *}$ & $0,59^{* *}$ & 0,86 & - \\
\hline 11 & $\begin{array}{l}\text { Furcht vor Misser- } \\
\text { folg (Sport) }\end{array}$ & 2,36 & 0,92 & $-0,27^{* *}$ & $-0,16^{*}$ & 0,02 & $-0,16^{*}$ & $-0,23^{* *}$ & $-0,08$ & $-0,41^{* *}$ & $-0,27^{* *}$ & $-0,60^{* *}$ & $-0,48^{* *}$ & 0,89 \\
\hline 12 & Spielklasse ${ }^{\mathrm{a}}$ & 2,83 & 1,24 & 0,06 & $0,21^{* *}$ & $-0,06$ & $0,23^{* *}$ & $-0,02$ & $-0,23^{* *}$ & 0,10 & $-0,01$ & $0,36^{* *}$ & 0,10 & $-0,22^{* *}$ \\
\hline
\end{tabular}

${ }^{\text {a} H o ̈ h e r e ~ K o d i e r u n g ~=~ b e s s e r e ~ L i g a: ~} 6=1$. Bundesliga, $5=2$. Bundesliga, $4=3$. Liga, $3=$ Regionalliga, $2=$ Oberliga, $1=$ tiefere Liga. $M=$ Mittelwert auf einer sechsstufigen Likert-Skala, $S D=$ Standardabweichung. Alle angegebenen Werte beziehen sich auf den Gesamtdatensatz. $N$ variiert wegen fehlender Werte bei $M$ und $S D$ zwischen 121 und 373 und bei den Korrelationen zwischen 62 und 373, da nicht in allen Teildatensätzen alle begleitenden Konstrukte erhoben wurden. Interne Konsistenzen (Cronbach's Alpha) der Konstrukte werden auf der Diagonalen berichtet $* p<0,05, * * p<0,01$

Abb. 1 Messmodell der Konfirmatorischen Faktorenanalyse (CFA) mit Faktorladungen auf Basis von Teildatensatz 2 (schematisch vereinfachte Darstellung)

Die angenommene Modellstruktur ${ }^{3}$ der acht Faktoren konnte somit bestätigt werden.

\subsection{Inhaltliche Beschreibung der KIPF-Dimensionen}

Die finale Version des KIPF enthält wie erwähnt acht Dimensionen, die im Folgenden inhaltlich näher beschrieben werden. Zudem erfolgt eine Zuordnung der Dimensionen zu den Kategorien des finalen Kompetenzstrukturmodells (vgl. Tab. 1) sowie zu den vier Handlungskompetenzbereichen nach Kauffeld (2006).

- Taktisches Wissen umfasst die Kenntnis von Fußballtaktiken sowie das fußballbezogene Fachwissen inklusive (mannschaftstaktischer) Feinheiten. Im Kompetenzstrukturmodell ist ein gleichnamiger Aspekt im Bereich der Fachkompetenz vorhanden.

- Wissen zu Ernährung und Gesundheit beinhaltet Kenntnisse darüber, welche Auswirkungen die Ernährung auf die Gesundheit und körperliche Leistungsfähigkeit hat. Auch diese Kompetenzdimension ist der Fachkompetenz zugeordnet und nennt sich im Kompetenzstrukturmodell Wissen zu Gesundheit und Lebensführung.

- Einsatz von Resilienzmethoden umfasst die Anwendung von Methoden zur Zielfokussierung, zur Aufgabenpriorisierung und zum Umgang mit Stress. Diese Dimension kann den Aspekten Methoden zur Fokussierung und zum Umgang mit Stress sowie Zielorientiertheit des Handelns/Zielsetzung im Kompetenzstrukturmodell zugeordnet werden und fällt damit in den Bereich der Methodenkompetenz.

- Umgang mit Medien beschreibt die Expertise der Fußballspieler, sich gegenüber den Medien angemessen zu verhalten und sich in öffentlichen Interviews souverän zu präsentieren. Der gleichnamige Aspekt im Kompetenzstrukturmodell ist ebenfalls dem Bereich Methodenkompetenz zugeordnet.

- Kommunikationsfähigkeit beinhaltet das Lösen von Problemen und Schwierigkeiten im Umgang mit Mitspielern

\footnotetext{
${ }^{3}$ Zusätzlich wurden zwei alternative Modelle untersucht. Zum einen wurde ein Modell mit einem Globalfaktor berechnet, auf den die acht Kompetenzfaktoren laden. Dieses Modell wies jedoch eine geringere Modellgüte auf als das Modell mit den kovariierend in Faktoren, $\chi^{2} / \mathrm{df}=385,552 / 244=1,58, \quad p<0,001 ; \quad \mathrm{CFI}=0,915 ; \quad \mathrm{SRMR}=0,100 ;$ RMSEA $=0,056,90 \% \mathrm{CI}=[0,045,0,066]$. Außerdem zeigte sich bei Item TA2 eine negative Varianz, was ein Anzeichen für eine Missspezifikation bezüglich der angenommenen Modellstruktur sein kann.

Zum anderen wurde ein Modell höherer Ordnung berechnet, in dem jeweils zwei Kompetenzen auf einen übergeordneten Faktor laden (z. B. Mentale Stärke und Kritikfähigkeit auf den Faktor Selbstkompetenz, vgl. Abb. 1). Dieses Modell konvergierte nicht bei der Berechnung, sodass kein Modellfit angegeben werden kann - dies spricht ebenfalls für eine Missspezifikation bezüglich der angenommenen Modellstruktur und/oder eine (zu) geringe Stichprobengröße in Verbindung mit einer (zu) hohen Modellkomplexität (vgl. Kline 2016).
}

durch Einsatz von angemessener Kommunikation. Im Kompetenzstrukturmodell ist ein gleichnamiger Aspekt im Bereich der Sozialkompetenz vorhanden.

- Teamunterstützende Anpassungsfähigkeit deckt das Verhalten $a b$, die eigene Mannschaft zu unterstützen, die Entscheidungen des Trainers zu respektieren und sich somit im Sinne des Teamerfolgs sozial angepasst zu verhalten. Diese Dimension kann den Aspekten Teamunterstützung sowie Soziale Anpassungsfähigkeit und Akzeptanz im Kompetenzstrukturmodell zugeordnet werden und fällt damit in den Bereich der Sozialkompetenz.

- Mentale Stärke umfasst den positiven Umgang mit Leistungsdruck, Selbstzweifeln und Nervosität - im Gegensatz zur Dimension Einsatz von Resilienzmethoden beziehen sich die Items dieser Dimension auf den Zeitraum während des Fußballspiels. Der gleichnamige Aspekt im Kompetenzstrukturmodell ist dem Bereich Selbstkompetenz zugeordnet; zudem kann der Aspekt Selbstbewusstsein mit dieser Dimension in Verbindung gebracht werden.

- Kritikfähigkeit beschreibt den positiven Umgang mit Kritik, um daraus zu lernen und die eigene Spielweise zu verbessern. Im Kompetenzstrukturmodell ist ein gleichnamiger Aspekt ebenfalls im Bereich der Selbstkompetenz vorhanden; darüber hinaus umfasst diese Dimension auch den Aspekt Reflexionsfähigkeit.

\section{4 Überprüfung der Validität des KIPF}

Moosbrugger und Kelava (2012) empfehlen die Untersuchung folgender Validitätsaspekte, ,[u]m ein differenziertes Bild der Gültigkeit eines Tests zu erhalten“ (S. 15): 1) Inhaltsvalidität, 2) Augenscheinvalidität, 3) Konstruktvalidität, 4) Kriteriumsvalidität.

Die praxisnahe Entwicklungsweise des KIPF - von der Aufstellung des Kompetenzstrukturmodells bis zur Erstellung des Itempools - unter Einbezug verschiedener Experten aus dem Fußballsport und der Wissenschaft legt eine hohe inhaltliche Validität nahe (vgl. Moosbrugger und Kelava 2012). Die Formulierungen der Items beziehen sich inhaltlich direkt auf den Arbeitsalltag von professionellen Fußballspielern. Auch die durch die EFA abgeleiteten Faktoren entsprechen den beruflichen Anforderungen, die an Profifußballspieler gestellt werden.

Im Folgenden wird ausführlich die Überprüfung der Konstruktvalidität berichtet. Evidenzen zur Augenscheinvalidität sowie zur Kriteriumsvalidität wurden in der vorliegenden Studie noch nicht erbracht. Auf diese Aspekte wird im Abschnitt Stärken, Limitationen und weiterer Forschungsbedarf eingegangen. 


\subsection{Aufstellung der Hypothesen zur Konstruktvalidierung}

Zur Überprüfung der Konstruktvalidität wurden Hypothesen zu den Zusammenhängen der Kompetenzen des KIPF mit konzeptionell nahestehenden Konstrukten formuliert. In der bisherigen Forschung wurde Performanz, als dessen Vorbedingung die Kompetenz gilt, mit Selbstwirksamkeit und Leistungsmotivation in Verbindung gebracht. Diese Konstrukte stehen daher im Fokus der Hypothesenentwicklung zur Konstruktvalidierung.

Selbstwirksamkeit ist definiert als das Vertrauen einer Person in die eigene Fähigkeit, schwierige Probleme zu bewältigen - mit anderen Worten, der Glaube an die Befähigung, eine Aufgabenstellung erfolgreich zu meistern (Bandura 1977; Rigotti et al. 2008). Das Konstrukt Selbstwirksamkeit gilt als eines der einflussreichsten, wenn es darum geht, das Streben nach Performanz im Sport zu erklären - seit den 1980er-Jahren wurden zahlreiche Studien dazu durchgeführt (für einen Überblick über die frühen Studien siehe Feltz und Lirgg 2001). Moritz et al. (2000) zeigten zudem meta-analytisch einen durchschnittlichen $\mathrm{Zu}$ sammenhang zwischen Selbstwirksamkeit und sportlicher Performanz von $r=0,38$ auf. Im Bereich Jugendfußball finden sich auch spätere Studien, die geringe bis mittlere positive Zusammenhänge zwischen Selbstwirksamkeit und Performanz aufzeigen (Bray et al. 2004; García-Naveira 2018; Höner und Feichtinger 2016).

Bezogen auf die Selbstwirksamkeit sind sportliche Höchstleister nicht nur in ihrem physischen Können effektiver, sondern vertrauen auch stärker auf ihre kognitiven Selbstregulationsfähigkeiten (z.B. Veränderungen in Spielsituationen erkennen, wirksame Handlungsstrategien wählen, mögliche Handlungen des Gegenspielers voraussehen, reaktionsschnelle Entscheidungen zu treffen, mit hohem Druck sowie Rückschlägen und Ablenkungen umgehen können; Beilock und Feltz 2007, S. 160f.). Daher ist anzunehmen, dass Selbstwirksamkeit auch mit den Kompetenzfacetten des KIPF in Zusammenhang steht.

Hypothese 1 Profifußball-Kompetenz hängt positiv zusammen mit fußballbezogener Selbstwirksamkeit.

Das Konstrukt der Leistungsmotivation bezieht sich darauf, ob eine Person eine leistungsbezogene Situation eher aufsucht oder sie meidet (Atkinson 1957; Brunstein und Heckhausen 2018). Hoffnung auf Erfolg zeigt sich dabei durch Zuversicht in leistungsorientierten Situationen und geht mit einer positiven Bewertung der eigenen Fähigkeiten einher (Kleinbeck und Kleinbeck 2009) - Furcht vor Misserfolg ergibt sich aus der Angst zu scheitern, der Zuschreibung eigener Unfähigkeit, oder aus angelernten negativen sozialen Konsequenzen im Zusammenhang mit derartigen Misserfolgen (Langens 2009). Diese beiden voneinander weitestgehend unabhängigen Facetten der Leistungsmotivation wurden in vielen Studien mit Performanz in Verbindung gebracht - auch im Fußballbereich, wo sich positive Zusammenhänge von Hoffnung auf Erfolg sowie negative Zusammenhänge von Furcht vor Misserfolg jeweils mit verschiedenen Performanzvariablen ergaben (Coetzee et al. 2006; Höner und Feichtinger 2016; Murr et al. 2018a; Sagar et al. 2010; Zuber und Conzelmann 2014; Zuber et al. 2015). Da Kompetenz die Voraussetzung von Performanz ist, ist davon auszugehen, dass auch die KIPF-Dimensionen mit den Facetten der Leistungsmotivation entsprechend zusammenhängen.

Hypothese 2a Profifußball-Kompetenz hängt positiv zusammen mit Hoffnung auf Erfolg (Leistungsmotivation).

Hypothese 2b Profifußball-Kompetenz hängt negativ zusammen mit Furcht vor Misserfolg (Leistungsmotivation).

Höhere Spielklassen im Fußball weisen ein höheres Professionalitätsniveau auf als niedrigere, was sich anhand von Kriterien wie dem erzielten Umsatz der Sportvereine und der medialen Präsenz ablesen lässt (Fahrner 2012). Dies geht auch mit höheren Kompetenzanforderungen an die Profifußballspieler einher. Gledhill et al. (2017) sprechen beispielsweise davon, dass anhand der Ausprägung von psychosozialen Faktoren zwischen verschiedenen Performanzniveaus im Fußball differenziert werden kann bzw. diese Faktoren mit dem Karrierefortschritt und dem persönlichen Aufstieg auf ein höheres Niveau in Zusammenhang stehen. Es ist folglich anzunehmen, dass die Kompetenzen bei Spielern in einer höheren Spielklasse ebenfalls eine höhere Ausprägung aufweisen.

Hypothese 3 Profifußball-Kompetenz hängt positiv zusammen mit der Spielklassenhöhe.

\section{2 Überprüfung der Hypothesen zur Konstruktvalidierung}

Um die aufgestellten Hypothesen überprüfen zu können, wurden die genannten nahestehenden Konstrukte in den Fragebogen integriert, den die Fußballspieler ausfüllten. Das nahestehende Konstrukt fußballbezogene Selbstwirksamkeit wurde mit einer aus sechs Items bestehenden Kurzskala gemessen (Gerlach 2004; $\alpha=0,80$, Beispielitem: „Auch wenn ich einen guten Gegenspieler habe, glaube ich, dass ich ihm trotzdem mein Spiel aufzwingen kann."). Die Leistungsmotivation wurden mit einer Kurzskala von Wendland et al. (2003) erhoben (vgl. auch Elbe et al. 2005; Wenhold et al. 2009), wobei beide Komponenten je fünf Items umfassten: Hoffnung auf Erfolg $(\alpha=0,86$, Beispielitem: „Wenn mir beim Fußball eine Herausforderung gestellt wird, die ich möglicherweise lösen kann, 
dann reizt es mich, diese sofort in Angriff zu nehmen.") und Furcht vor Misserfolg ( $\alpha=0,89$, Beispielitem: ,Schon die Vorstellung, beim Fußball vor eine neue unbekannte Herausforderung gestellt $\mathrm{zu}$ werden, macht mich etwas ängstlich.“). Die Abfrage der Spielklassenhöhe erfolgte mit einem Item mit Auswahloptionen $\mathrm{zu}$ den Ligen von der 1. Bundesliga bis zur 5. Liga (Oberliga) und tieferen, sonstigen Ligen. Aus testökonomischen Gründen wurden die nahestehenden Konstrukte nur in den Fragebögen für zwei (Selbstwirksamkeit) bzw. vier (Leistungsmotivation) der fünf Akquiserunden integriert, sodass sich ein variierendes $N$ für diese Konstrukte ergab (vgl. Abb. 4). Die Spielklassenhöhe hingegen wurde in allen eingesetzten Fragebögen abgefragt.

Tab. 4 zeigt die Ergebnisse der Korrelationsanalyse, mit deren Hilfe die Zusammenhänge zwischen den Konstrukten auf Grundlage des Gesamtdatensatzes berechnet wurden. Hypothesenkonform ergaben sich mittlere positive, signifikante Zusammenhänge zwischen der fußballbezogenen Selbstwirksamkeit und sieben der acht Kompetenzdimensionen ( $r$ zwischen 0,25 und 0,44). Lediglich die Dimension Teamunterstützende Anpassungsfähigkeit ist nicht signifikant mit Selbstwirksamkeit verbunden. Dies könnte daran liegen, dass Spieler die Entscheidungen ihres Trainers respektieren und ihre Mannschaft unterstützen können, wenn sie nicht spielen - auch völlig unabhängig von dem Zutrauen in ihre eigene Stärke. Hypothese 1 konnte somit größtenteils bestätigt werden. Hoffnung auf Erfolg korreliert ebenfalls positiv mit sechs der acht Kompetenzdimensionen ( $r$ zwischen 0,16 und 0,32). Wie auch bei der Selbstwirksamkeit findet sich der größte Zusammenhang mit der Dimension Taktisches Wissen. Keine signifikanten Zusammenhänge konnten zwischen Hoffnung auf Erfolg und Umgang mit Medien sowie erneut Teamunterstützende Anpassungsfähigkeit gefunden werden. Eine Erklärung könnte sein, dass beide Dimensionen nicht direkt die eigene sportliche Leistung betreffen, auch wenn sie als Bedingungen für den langfristigen sportlichen Erfolg bedeutend sind. Hypothese 2a konnte daher teilweise bestätigt werden. Furcht vor Misserfolg korreliert wie erwartet negativ mit der Kompetenz - hier zeigten sich signifikante $\mathrm{Zu}$ sammenhänge mit sechs der acht Kompetenzdimensionen ( $r$ zwischen -0,16 und -0,41). Keine signifikanten Verbindungen ergaben sich mit den Dimensionen Einsatz von Resilienzmethoden sowie Teamunterstützende Anpassungsfähigkeit. Diese Kompetenzen scheinen relativ unabhängig davon, ob sich jemand vor sportlichen Herausforderungen fürchtet oder diese meidet, bei den Spielern vorhanden $\mathrm{zu}$ sein. Hypothese $2 \mathrm{~b}$ konnte damit ebenfalls teilweise bestätigt werden.

Die Spielklassenhöhe korreliert signifikant moderat positiv mit dem Wissen zu Ernährung und Gesundheit $(r=0,21)$ und dem Umgang mit Medien $(r=0,23)$. Diese Befunde sind insofern hypothesenkonform, als dass ein höheres Niveau an Professionalität in der Spielklasse einen ebenfalls professionelleren Lebenswandel der Spieler sowie Auftritt in der Öffentlichkeit erfordert. Teamunterstützende Anpassungsfähigkeit korreliert negativ mit der Spielklassenhöhe ( $r=-0,23$ ) - dies lässt die Schlussfolgerung zu, dass Spieler in höheren Spielklassen mehr auf ihren eigenen Erfolg achten und die Teamunterstützung geringer ausfällt. Mit den anderen fünf Kompetenzdimensionen ergeben sich keine signifikanten Zusammenhänge, sodass Hypothese 3 größtenteils nicht bestätigt werden konnte.

\subsection{Konvergente und diskriminante Validität}

Zur Konstruktvalidität zählt gemäß Moosbrugger und Kelava (2012) ebenfalls die konvergente und diskriminante Validität. Zur Bestimmung dieser Validitäten bei den acht Faktoren des KIPF wurden die durchschnittlich erfasste Varianz (auch bekannt als average variance extracted, AVE) für jeden Faktor und die gemeinsame Varianz zwischen den Faktoren untereinander (shared variance, $S V$ ) berechnet. Ein AVE-Wert >0,50 gilt als Indiz für konvergente Validität (Hair et al. 2010). AVE-Werte, die größer als die SV-Werte zwischen zwei Faktoren sind, sprechen für diskriminante Validität (Farrell 2010).

Der AVE-Wert liegt bei dem Konstrukt Mentale Stärke bei 0,38, sowie bei dem Konstrukt Teamunterstützende Anpassungsfähigkeit bei 0,49, ansonsten bei allen Konstrukten $>0,50(0,54$ bis 0,76), sodass teilweise Evidenz für konvergente Validität vorliegt. Die SV-Werte liegen zwischen 0,00 und 0,17 und somit stets unter den AVE-Werten; daher kann von diskriminanter Validität ausgegangen werden. Die größte gemeinsame Varianz teilen die Konstrukte Einsatz von Resilienzmethoden und Wissen zu Ernährung und Gesundheit $(\mathrm{SV}=0,17)$.

\subsection{Kompetenzdimensionen und Spielpositionen}

Darüber hinaus wurde explorativ untersucht, ob sich die Kompetenzausprägungen der Fußballspieler auf verschiedenen Spielpositionen unterscheiden. Bei 190 der 373 Befragungsteilnehmer war die Spielposition - erhoben mit vier Ausprägungen - bekannt (12,6\% Tor, 32,1\% Abwehr, $37,9 \%$ Mittelfeld, 17,4\% Angriff). Eine mit der Statistiksoftware SPSS 25 durchgeführte einfaktorielle ANOVA ergab keine signifikanten Unterschiede zwischen den vier Spielpositionsgruppen bei keiner der acht Kompetenzdimensionen; die $p$-Werte lagen zwischen 0,200 und 0,707. Daraus lässt sich folgern, dass das KIPF bei allen Profifußballspielern - unabhängig von deren Spielpositionen eingesetzt werden kann, da die Spieler auf unterschiedlichen Positionen ähnliche Ist-Werte bezüglich der Kompetenzausprägungen angeben. Ob ebenfalls keine Unterschie- 
de in Hinblick auf die acht Kompetenzdimensionen bei den Anforderungsprofilen (Soll-Werten) für verschiedene Spielpositionen existieren, könnte die zukünftige Forschung durch weitere Analysen (z. B. Expertenbefragungen) klären.

\section{Stärken, Limitationen und weiterer Forschungsbedarf}

Mit dem KIPF liegt ein neu entwickeltes Instrument zur Erfassung der beruflichen Kompetenzen professioneller Fußballspieler vor, welches die drei Hauptgütekriterien von psychometrischen Verfahren - Objektivität, Reliabilität, Validität - (Döring und Bortz 2016; Moosbrugger und Kelava 2012) überwiegend erfüllt:

Durch die skalenbasierte Auswertung ist die Objektivität bzw. Anwenderunabhängigkeit größtenteils sichergestellt zumindest, was die Auswertungsobjektivität angeht (vgl. Döring und Bortz 2016). Die Entwicklung eines Testmanuals könnte zukünftig auch die Durchführungs- und Interpretationsobjektivität gewährleisten, um Fehlanwendungen des KIPF in der Praxis zu vermeiden. Die Bestimmung der Reliabilität erfolgte durch die Überprüfung der internen Konsistenz der Konstrukte; die Werte für Cronbach's Alpha liegen - wie oben beschrieben - insgesamt in einem annehmbaren bis zufriedenstellenden Bereich, wenngleich das Konstrukt Mentale Stärke im Gesamtdatensatz einen eher schwächeren Wert aufweist. Bezüglich der Validität wurden die Inhaltsvalidität sowie die Konstruktvalidität bereits geprüft (vgl. Abschnitt Überprüfung der Validität des KIPF). Um Evidenz für die Augenscheinvalidität des KIPF zu erbringen, könnte in einem weiteren Validierungsschritt mit Spielerinterviews untersucht werden, inwiefern die Zielgruppe der Fußballspieler die Zuordnung der Items zu den Faktoren inhaltlich nachvollziehbar findet. Eine hohe Augenscheinvalidität steigert die Akzeptanz des Instruments bei den Testpersonen, allerdings auch die Verfälschbarkeit des Tests (Döring und Bortz 2016), da die Testpersonen die latente Teststruktur durchschauen. Weitere Studien könnten sich zudem der Überprüfung der Kriteriumsvalidität annehmen (vgl. Moosbrugger und Kelava 2012). Hierbei könnten zur Prüfung der konkurrenten Validität die Kompetenzen mit aktuellen oder retrospektiven Leistungsdaten bzw. Erfolgskriterien der Fußballspieler in Verbindung gebracht werden (für Möglichkeiten zur Operationalisierung von Erfolg im Profifußball siehe Decius und Erdmann 2017). Zur Prüfung der prognostischen Validität bietet sich ein längsschnittliches Studiendesign an, um die Kompetenz- und Leistungsentwicklung über mehrere Jahre betrachten zu können.

Bei der Korrelationsanalyse zur Überprüfung der Konstruktvalidität fiel auf, dass die Kompetenzdimension Teamunterstützende Anpassungsfähigkeit als einzige Dimension keinen signifikanten Zusammenhang mit allen drei nahestehenden Validierungskonstrukten aufwies. Mit der Höhe der Spielklasse ergab sich hingegen sogar eine signifikante negative Korrelation. Dies wirft die Frage auf, ob Teamunterstützende Anpassungsfähigkeit als Kompetenz für den Profifußball eine Rolle spielt. Allerdings wurde dieser Kompetenzaspekt häufig in den Spieler- und Experteninterviews genannt (vgl. Tab. 5 im Anhang). Zukünftige Studien könnten untersuchen, ob Anpassungsfähigkeit eine zwar gewünschte Sollkompetenz ist, die vorhandene Ausprägung für die Performanz der Fußballspieler jedoch eventuell eine nur geringe oder keine Bedeutung hat.

Bezüglich der verwendeten Daten zur Entwicklung des KIPF müssen folgende Einschränkungen berücksichtigt werden: Die Fußballspieler in der Stichprobe, bei denen das Alter bekannt war, wiesen ein geringes Durchschnittsalter von 21 Jahren bei einer Standardabweichung von 6 Jahren auf ${ }^{4}$. Auch wenn das Leistungsalter professioneller Fußballspieler deutlich geringer ist als in anderen Berufsgruppen, ist fraglich, ob die Altersstruktur der Stichprobe die Grundgesamtheit der professionellen Fußballspieler in Deutschland adäquat abbildet. Dies sollte in Folgestudien berücksichtigt werden. Zudem wurden ausschließlich empirische Daten von männlichen Fußballspielern herangezogen, da etwa $90 \%$ aller weltweit fußballspielenden Personen männlich sind (Haugaasen und Jordet 2012) und der männliche Fußballsport sich auch bei den Fans großer Beliebtheit erfreut. Nichtsdestotrotz könnte die zukünftige Forschung eine Übertragung des KIPF auf den weiblichen Fußballsport in Betracht ziehen und überprüfen, ob die gleichen Items hierfür valide und reliabel sind, oder ob Anpassungen erforderlich sind. Zudem wurden nur Fußballspieler in Deutschland in die Stichprobe aufgenommen. Dellal et al. (2011) fanden in ihrer Studie teilweise Unterschiede bei physischen und technischen Leistungsvariablen von Fußballspielern in der englischen Premier League sowie der spanischen Primera División. Es ist nicht ausgeschlossen, dass es interkulturelle Unterschiede auch bei beruflichen Kompetenzen von Fußballspielern in verschiedenen Ländern gibt, auch wenn diese wegen der relativen Einfachheit und Universalität des Fußballsports gering ausfallen dürften. Nichtsdestotrotz könnten sich zukünftige Studien eines Kulturvergleichs annehmen und dazu das KIPF in andere Sprachen übertragen.

Obwohl ein Kompetenzinventar für den Profifußball entwickelt wurde, war es zur Generierung einer angemessen großen Stichprobe erforderlich, auch semi-professionelle

\footnotetext{
${ }^{4}$ Allerdings ist zu berücksichtigen, dass das Alter bei lediglich einem Drittel der Stichprobe bekannt war. Eigene Eindrücke bei der Akquise und Gespräche mit den Vereinsverantwortlichen ergaben, dass das Alter der teilnehmenden Spieler in den Teilstichproben, bei denen keine Altersangabe vorliegt, deutlich über dem Durchschnitt von 21 Jahren lag.
} 
Fußballspieler bei der Akquise zu berücksichtigen, was den hohen Anteil an Regional- und Oberligaspielern in der Stichprobe erklärt. Die Heterogenität bezüglich der Professionalität der Stichprobenteilnehmer erschwert daher die Ableitung allgemeingültige Aussagen. Folge Untersuchungen könnten sich mit der Frage beschäftigen, welche der acht Kompetenzen auch für den Amateursport relevant sind, und welche lediglich im Profibereich eine wichtige Rolle spielen; erste Hinweise ergaben sich in der vorliegenden Studie durch die Korrelationen der Spielklassenhöhe mit den Kompetenzen Wissen zu Ernährung und Gesundheit und Umgang mit Medien. Die Stichprobengröße ist - begründet in der schwierigen Verfügbarkeit der Zielgruppe - insgesamt als eher gering einzuschätzen. Zukünftige Studien könnten eine Kooperation mit Fußballvereinen anstreben, um den Zugang zu den Profifußballspielern zu verbessern und das Commitment für die Teilnahme der Spieler zu erhöhen.

Da das KIPF keine physischen oder technischen Fertigkeiten und Fähigkeiten umfasst, sondern die berufliche Handlungskompetenz misst, könnte das Inventar möglicherweise auch für den Einsatz bei anderen Mannschaftssportarten angepasst werden, in denen ähnliche psychologische Anforderungen an die Sportler gestellt werden, beispielsweise in den Bereichen Umgang mit Medien, Mentale Stärke oder Einsatz von Resilienzmethoden. Zu nennen sind hier insbesondere die Sportarten Handball, Eishockey und Basketball, deren Profiligen in Deutschland als die erlösstärksten Sportligen nach dem Fußballbereich gelten (Vogel und Ehemann 2012).

Die vorliegende Studie untersuchte zudem ausschließlich die individuellen Handlungskompetenzen der Spieler. Zukünftig könnte betrachtet werden, inwieweit eine bestimmte Kompetenzstruktur auf Teamebene die Gesamtperformanz der Mannschaft beeinflusst. Möglicherweise ist eine hohe Ausprägung in einer oder mehreren Kompetenzdimensionen in Kombination betrachtet wichtiger für den Mannschaftserfolg als eine hohe Ausprägung in anderen Dimensionen.

\section{Fazit}

Mit Hilfe des Kompetenzinventars Profifußball (KIPF) können Anwenderinnen und Anwender in der Praxis die Ausprägung der beruflichen Handlungskompetenzen von Profifußballspielern ermitteln. Vereinsverantwortliche und insbesondere Trainer können das KIPF einsetzen, um einen Überblick über die vorhandenen Kompetenzen ihrer Spieler zu gewinnen und damit deren individuelle Performanzvoraussetzungen besser einschätzen zu können. Sportverbände können das KIPF zur Analyse von langfristigen Kompetenzprofil-Entwicklungen der Fußballer in ihrem Verbands- gebiet nutzen. Spielerberater können das KIPF verwenden, um die Stärken und Schwächen der von ihnen betreuten Spieler herauszufinden und ihnen Rückmeldungen zur individuellen Kompetenzentwicklung geben. Gleiches gilt für die Fußballspieler selbst, die das KIPF im Selbsttest bearbeiten können, um anhand des Ergebnisses über ihre Kompetenzausprägungen zu reflektieren.

In allen Anwendungsfällen kann das Ziel verfolgt werden, nach der Kompetenzdiagnose eine Förderung der vorhandenen Stärken und einen Ausgleich der Schwächen bei den Fußballspielern zu unterstützen. Ob sich aus den Ergebnissen des KIPF konkrete Kompetenzentwicklungsmaßnahmen ableiten lassen, sollte in zukünftigen Studien geprüft werden. Interessierte (Profi-)Fußballvereine können sich dazu gern beim Autor melden. Für eine einfache Handhabung in der Praxis wäre zudem eine technische Umsetzung in einem Online-Tool mit automatisierter Ergebnisdarstellung - möglichst in Echtzeit - bedenkenswert. Für die Wissenschaft bietet das KIPF die Möglichkeit, durch die Messung der Handlungskompetenzen die Rahmenbedingungen der Performanz weiter zu erforschen, insbesondere in Hinblick auf die Antezedenzien der Handlungskompetenz sowie zeitlich nachgelagerter Kompetenzentwicklungsergebnisse bei Profifußballspielern. 


\section{Anhang}

Tab. 5 Erweitertes, noch nicht finales Kompetenzstrukturmodell aus deduktiv abgeleiteten und induktiv ergänzten Kompetenzfacetten

\begin{tabular}{|c|c|c|c|c|c|}
\hline $\begin{array}{l}\text { Bereich der Hand- } \\
\text { lungskompetenz }\end{array}$ & Kompetenzfacetten & $\begin{array}{l}\text { Experten } \\
(\max .6)\end{array}$ & $\begin{array}{l}\text { Spieler (max. } \\
10)\end{array}$ & $\begin{array}{l}\text { Gesamt (max. } \\
16)\end{array}$ & $\begin{array}{l}\text { Relevanz (max. } \\
\text { 144) }\end{array}$ \\
\hline \multirow[t]{3}{*}{ Fachkompetenz } & $\begin{array}{l}\text { Mentale Fähigkeiten zur Anwendung } \\
\text { physischer Fertigkeiten (D) }\end{array}$ & 6 & 9 & 15 & 109 \\
\hline & Wissen/Fertigkeiten (D) & 1 & 8 & 9 & 58 \\
\hline & Spieltechniken (D) & 3 & 5 & 8 & 50 \\
\hline \multirow[t]{3}{*}{ Methodenkompetenz } & Fokussierung (D) & 6 & 10 & 16 & 107 \\
\hline & $\begin{array}{l}\text { Zielorientiertheit des Handelns/ } \\
\text { Zielsetzung (D) }\end{array}$ & 3 & 9 & 12 & 81 \\
\hline & Umgang mit Medien (I) & 4 & 4 & 8 & 62 \\
\hline \multirow[t]{8}{*}{ Sozialkompetenz } & Kommunikationsfähigkeit (D) & 5 & 10 & 15 & 110 \\
\hline & Respekt und Freundlichkeit zeigen (D) & 3 & 9 & 12 & 78 \\
\hline & Teamfähigkeit (D) & 3 & 6 & 10 & 71 \\
\hline & Soziale Anpassungsfähigkeit (D) & 4 & 6 & 10 & 64 \\
\hline & Wahrnehmung einer Vorbildfunktion (I) & 4 & 6 & 10 & 55 \\
\hline & Hilfsbereitschaft (I) & 3 & 4 & 7 & 47 \\
\hline & Akzeptanz fremder Entscheidungen (D) & 1 & 6 & 7 & 42 \\
\hline & Empathie (D) & 2 & 3 & 5 & 33 \\
\hline \multirow[t]{18}{*}{ Selbstkompetenz } & Selbstbewusstsein (D) & 4 & 10 & 14 & 96 \\
\hline & Disziplin (D) & 6 & 7 & 13 & 95 \\
\hline & Wille und Motivation (D) & 6 & 10 & 16 & 89 \\
\hline & $\begin{array}{l}\text { Umgang mit Stress/Leistung unter } \\
\text { Druck (D) }\end{array}$ & 5 & 7 & 12 & 87 \\
\hline & $\begin{array}{l}\text { Engagement und unbeirrte Karrierepla- } \\
\text { nung (D) }\end{array}$ & 5 & 7 & 12 & 85 \\
\hline & Durchhaltevermögen (I) & 4 & 9 & 13 & 83 \\
\hline & $\begin{array}{l}\text { Umgang mit Niederlagen und Rückschlä- } \\
\text { gen (D) }\end{array}$ & 6 & 3 & 9 & 68 \\
\hline & Reflexivität (D) & 5 & 5 & 10 & 66 \\
\hline & Realistische Selbsteinschätzung (D) & 4 & 8 & 12 & 57 \\
\hline & Verantwortungsbewusstsein (D) & 2 & 6 & 8 & 57 \\
\hline & Kritikfähigkeit (D) & 3 & 7 & 10 & 56 \\
\hline & Geduld (I) & 1 & 7 & 8 & 49 \\
\hline & Belastbarkeit (D) & 3 & 5 & 8 & 48 \\
\hline & Bodenständigkeit (I) & 5 & 3 & 8 & 48 \\
\hline & Emotionale Stabilität (D) & 4 & 2 & 6 & 38 \\
\hline & Lernwilligkeit (I) & 0 & 5 & 5 & 37 \\
\hline & Selbstständigkeit (D) & 5 & 3 & 8 & 34 \\
\hline & Positive Einstellung/Mentalität (D) & 0 & 3 & 3 & 19 \\
\hline
\end{tabular}

In der ersten und zweiten Zahlenspalte: Anzahl der Experteninterviews bzw. Spielerinterviews, in denen die Kompetenzfacette genannt wurde; in der dritten Zahlenspalte: Summe aus Experten- und Spielerinterviews; in der vierten Zahlenspalte: Relevanzwert für jede Kompetenzfacette, der aus drei Kriterien berechnet wurde (siehe Text)

$D$ Deduktiv aus der Literatur abgeleitet, $I$ Induktiv auf Basis der 16 Interviews ergänzt 
Tab. 6 Kovarianzen der Konfirmatorischen Faktorenanalyse (CFA)

\begin{tabular}{|c|c|c|}
\hline Kovarianz & Standardisiertes Beta & $p$-Wert \\
\hline \multicolumn{3}{|l|}{$\overline{\text { Resilienz } \sim ~}$} \\
\hline Lebensführung & 0,41 & 0,000 \\
\hline Taktikwissen & $-0,09$ & 0,301 \\
\hline Kommunikation & 0,20 & 0,025 \\
\hline Medienumgang & 0,18 & 0,034 \\
\hline Mentale Stärke & $-0,12$ & 0,216 \\
\hline Kritikfähigkeit & 0,19 & 0,053 \\
\hline Teamanpassung & $\mathbf{0 , 3 0}$ & 0,005 \\
\hline \multicolumn{3}{|l|}{ Lebensführung } \\
\hline Taktikwissen & 0,08 & 0,382 \\
\hline Kommunikation & 0,01 & 0,921 \\
\hline Medienumgang & 0,27 & 0,002 \\
\hline Mentale Stärke & $-0,05$ & 0,603 \\
\hline Kritikfähigkeit & 0,22 & 0,021 \\
\hline Teamanpassung & 0,02 & 0,796 \\
\hline \multicolumn{3}{|l|}{ Taktikwissen } \\
\hline Kommunikation & 0,25 & 0,010 \\
\hline Medienumgang & $\mathbf{0 , 3 9}$ & 0,000 \\
\hline Mentale Stärke & 0,27 & 0,043 \\
\hline Kritikfähigkeit & 0,00 & 0,981 \\
\hline Teamanpassung & 0,06 & 0,531 \\
\hline \multicolumn{3}{|c|}{ Kommunikation } \\
\hline Medienumgang & 0,22 & 0,013 \\
\hline Mentale Stärke & 0,28 & 0,015 \\
\hline Kritikfähigkeit & 0,25 & 0,006 \\
\hline Teamanpassung & 0,26 & 0,017 \\
\hline \multicolumn{3}{|c|}{ Medienumgang } \\
\hline Mentale Stärke & 0,26 & 0,019 \\
\hline Kritikfähigkeit & 0,04 & 0,655 \\
\hline Teamanpassung & $-0,06$ & 0,470 \\
\hline \multicolumn{3}{|l|}{ Mentale Stärke } \\
\hline Kritikfähigkeit & 0,06 & 0,574 \\
\hline Teamanpassung & 0,03 & 0,774 \\
\hline \multicolumn{3}{|l|}{ Kritikfähigkeit } \\
\hline Teamanpassung & 0,36 & 0,004 \\
\hline
\end{tabular}

$N=186$. Signifikante Kovarianzen, deren $p$-Wert $<0,05$ beträgt, sind mit Fettdruck hervorgehoben

Taktikwissen Taktisches Wissen, Lebensführung Wissen zu Ernährung und Gesundheit, Resilienz, Einsatz von Resilienzmethoden, Medienumgang Umgang mit Medien, Kommunikation Kommunikationsfähigkeit, Teamanpassung $=$ Teamunterstützende Anpassungsfähigkeit

Zusatzmaterial online Zusätzliche Informationen sind in der OnlineVersion dieses Artikels (https://doi.org/10.1007/s11612-021-00571-z) enthalten.

Danksagung Der Autor dankt Prof. Dr. Niclas Schaper (Universität Paderborn) für den fachlichen Austausch zum Kompetenzstrukturmodell und zum Itempool, sowie folgenden Personen für die Unterstützung bei der Literaturrecherche, Interviewdurchführungen, Transkriptionen der Interviews und der Teilnehmerakquise: André Brockschmidt, Sabrina Ernst, Felix Falke, Moritz Fritz, Moritz Kickermann,
Niklas Knüpp, Robin Köhler, Yannik Langesberg, Sebastian Marx, Mark Möbus, Sarah Richter, Pascal Schmeing.

Funding Open Access funding enabled and organized by Projekt DEAL.

Open Access Dieser Artikel wird unter der Creative Commons Namensnennung 4.0 International Lizenz veröffentlicht, welche die Nutzung, Vervielfältigung, Bearbeitung, Verbreitung und Wiedergabe in jeglichem Medium und Format erlaubt, sofern Sie den/die ursprünglichen Autor(en) und die Quelle ordnungsgemäß nennen, einen Link zur Creative Commons Lizenz beifügen und angeben, ob Änderungen vorgenommen wurden.

Die in diesem Artikel enthaltenen Bilder und sonstiges Drittmaterial unterliegen ebenfalls der genannten Creative Commons Lizenz, sofern sich aus der Abbildungslegende nichts anderes ergibt. Sofern das betreffende Material nicht unter der genannten Creative Commons Lizenz steht und die betreffende Handlung nicht nach gesetzlichen Vorschriften erlaubt ist, ist für die oben aufgeführten Weiterverwendungen des Materials die Einwilligung des jeweiligen Rechteinhabers einzuholen.

Weitere Details zur Lizenz entnehmen Sie bitte der Lizenzinformation auf http://creativecommons.org/licenses/by/4.0/deed.de.

\section{Literatur}

Ali, A. (2011). Measuring soccer skill performance: a review. Scandinavian Journal of Medicine \& Science in Sports, 21(2), 170-183. https://doi.org/10.1111/j.1600-0838.2010.01256.x.

Ambe, H., Matsumoto, D., Sakamoto, E., Murase, K., \& Ochiai, M. (2013). Development of a self-perceived soccer competence scale. Football Science, 10, 18-27.

Apitzsch, T. (2012). Kompetenzprofile von Trainern und Sportmanagern im Leistungssport (Dissertationsschrift). Köln: Deutsche Sporthochschule

Atkinson, J.W. (1957). Motivational determinants of risk-taking behavior. Psychological Review, 64(6, Pt.1), 359-372. https://doi. org/10.1037/h0043445.

Bandura, A. (1977). Self-efficacy: toward a unifying theory of behavioral change. Psychological review, 84(2), 191-215. https://doi. org/10.1037/0033-295x.84.2.191.

Beilock, S. L., \& Feltz, D. L. (2007). Selbstwirksamkeit und Expertise. In N. Hagemann, M. Tietjens \& B. Strauß (Hrsg.), Psychologie der sportlichen Höchstleistung. Grundlagen und Anwendungen der Expertiseforschung im Sport (S. 156-174). Göttingen: Hogrefe.

Bray, S. R., Balaguer, I., \& Duda, J. L. (2004). The relationship of task self-efficacy and role efficacy beliefs to role performance in Spanish youth soccer. Journal of sports sciences, 22(5), 429-437. https://doi.org/10.1080/02640410410001675333.

Brunstein, J.C., \& Heckhausen, H. (2018). Leistungsmotivation. In J. Heckhausen \& H. Heckhausen (Hrsg.), Motivation und Handeln (S. 163-221). Berlin, Heidelberg, New York: Springer. https://doi.org/10.1007/978-3-642-12693-2_6.

Coetzee, B., Grobbelaar, H.W., \& Gird, C. C. (2006). Sport psychological skills that distinguish successful from less successful soccer teams. Journal of Human Movement Studies, 51(6), 383-402.

Conway, J.M., \& Huffcutt, A.I. (2003). A review and evaluation of exploratory factor analysis practices in organizational research. Organizational research methods, 6(2), 147-168. https://doi.org/ 10.1177/1094428103251541.

Cook, C., Crust, L., Littlewood, M., Nesti, M., \& Allen-Collinson, J. (2014). 'What it takes': perceptions of mental toughness and its development in an English Premier League Soccer Academy. 
Qualitative research in sport, exercise and health, 6(3), 329-347. https://doi.org/10.1080/2159676x.2013.857708.

Costello, A. B., \& Osborne, J. (2005). Best practices in exploratory factor analysis: four recommendations for getting the most from your analysis. Practical Assessment Research \& Evaluation, 10(7), 1-9. https://doi.org/10.4135/9781412995627.d8.

Decius, J. (2018). Erfolg bei Profi-Fußballspielern - Welche Rolle spielen Glück und Pech im Karriereverlauf? In Blitzlicht-Beitrag präsentiert auf dem DGPS-Kongress, 18.09.2018, Frankfurt. https://doi.org/10.13140/RG.2.2.20537.52327.

Decius, J., \& Erdmann, N. (2017). Berufsfeld Profifußball - Wie lässt sich der Karriereerfolg von professionellen Fußballspielern messbar machen? In Beitrag präsentiert auf der 10. Tagung der Fachgruppe AOW der DGPs, 14.09.2017, Dresden. https://doi.org/10. 13140/RG.2.2.30603.85281.

Decius, J., Schaper, N., \& Seifert, A. (2019). Informal workplace learning: Development and validation of a measure. Human Resource Development Quarterly, 30(4), 495-535. https://doi.org/10.1002/ hrdq. 21368.

Dellal, A., Chamari, K., Wong, D. P., Ahmaidi, S., Keller, D., Barros, R., \& Carling, C. (2011). Comparison of physical and technical performance in European soccer match-play: FA Premier League and La Liga. European Journal of Sport Science, 11(1), 51-59. https://doi.org/10.1080/17461391.2010.481334.

DeVellis, R.F. (2003). Scale development. Theory and applications (2. Aufl.). Thousand Oaks: SAGE.

Döring, N., \& Bortz, J. (2016). Forschungsmethoden und Evaluation in den Sozial- und Humanwissenschaften. Berlin, Heidelberg, New York: Springer. https://doi.org/10.1007/978-3-642-41089-5.

Elbe, A. M., Wenhold, F., \& Müller, D. (2005). Zur Reliabilität und Validität der Achievement Motives Scale-Sport. Zeitschrift für Sportpsychologie, 12(2), 57-68. https://doi.org/10.1026/16125010.12.2.57.

Fahrner, M. (2014). Grundlagen des Sportmanagements. München: De Gruyter. https://doi.org/10.1524/9783486989410.

Farrell, A. M. (2010). Insufficient discriminant validity: A comment on Bove, Pervan, Beatty, and Shiu (2009). Journal of Business Research, 63(3), 324-327. https://doi.org/10.1016/j.jbusres.2009. 05.003.

Feichtinger, P., \& Höner, O. (2015). Talented football players' development of achievement motives, volitional components, and selfreferential cognitions: A longitudinal study. European journal of sport science, 15(8), 748-756. https://doi.org/10.1080/17461391. 2015.1051134.

Feltz, D.L., \& Lirgg, C.D. (2001). Self-efficacy beliefs of athletes, teams, and coaches. In R. N. Singer, H. A. Hausenblas \& C. M. Janelle (Hrsg.), Handbook of sport psychology (S. 340-361). New York: Wiley.

Flanagan, J. C. (1954). The critical incident technique. Psychological bulletin, 51(4), 327-360. https://doi.org/10.1037/h0061470.

Forsman, H., Blomqvist, M., Davids, K., Liukkonen, J., \& Konttinen, N. (2016)a. Identifying technical, physiological, tactical and psychological characteristics that contribute to career progression in soccer. International Journal of Sports Science \& Coaching, 11(4), 505-513. https://doi.org/10.1177/1747954116655051.

Forsman, H., Gråstén, A., Blomqvist, M., Davids, K., Liukkonen, J., \& Konttinen, N. (2016)b. Development and validation of the perceived game-specific soccer competence scale. Journal of sports sciences, 34(14), 1319-1327. https://doi.org/10.1080/02640414. 2015.1125518.

Franke, G. (2005). Facetten der Kompetenzentwicklung. Bielefeld: Bertelsmann.

García-Naveira, V. (2018). Self-efficacy and performance in soccer players. Cuadernos De Psicología Del Deporte, 18(2), 68-79.

Gerlach, E. (2004). Selbstwirksamkeitserwartung im Fußball. Entwicklung eines neuen Messinstruments. In R. Naul (Hrsg.), Nachwuchsförderung im Fußballsport - Neue Wege in Deutsch- land und Europa. Beiträge und Analysen zum Fußballsport XIV (S. 212-227). Aachen: Meyer \& Meyer.

Gledhill, A., Harwood, C., \& Forsdyke, D. (2017). Psychosocial factors associated with talent development in football: A systematic review. Psychology of Sport and Exercise, 31, 93-112. https://doi. org/10.1016/j.psychsport.2017.04.002.

Gould, D., Dieffenbach, K., \& Moffett, A. (2002). Psychological characteristics and their development in Olympic champions. Journal of Applied Sport Psychology, 14(3), 172-204. https://doi.org/10. 1080/10413200290103482.

Hair, J. F., Black, W.C., Babin, B.J., \& Anderson, R.E. (2010). Multivariate data analysis: a global perspective (3. Aufl.). Harlow: Pearson.

Hartig, J., \& Klieme, E. (2006). Kompetenz und Kompetenzdiagnostik. In K. Schweizer (Hrsg.), Leistung und Leistungsdiagnostik (S. 127-143). Berlin, Heidelberg, New York: Springer. https://doi. org/10.1007/3-540-33020-8_9.

Haugaasen, M., \& Jordet, G. (2012). Developing football expertise: a football-specific research review. International Review of Sport and Exercise Psychology, 5(2), 177-201. https://doi.org/10.1080/ 1750984x.2012.677951.

Haupt, T., \& Schöttl, K. (2016). Social Media Marketing im Spitzensport. In A. Schneider, J. Köhler \& F. Schumann (Hrsg.), Sport im Spannungsfeld zwischen Medien und Psychologie: Aktuelle Herausforderungen und Perspektiven (S. 109-136). Wiesbaden: Springer. https://doi.org/10.1007/978-3-658-11579-1_5.

Holt, N. L., \& Dunn, J. G. (2004). Toward a grounded theory of the psychosocial competencies and environmental conditions associated with soccer success. Journal of applied sport psychology, 16(3), 199-219. https://doi.org/10.1080/10413200490437949.

Höner, O., \& Feichtinger, P. (2016). Psychological talent predictors in early adolescence and their empirical relationship with current and future performance in soccer. Psychology of Sport and Exercise, 25, 17-26. https://doi.org/10.1016/j.psychsport.2016.03.004.

Horn, J.L. (1965). A rationale and test for the number of factors in factor analysis. Psychometrika, 30(2), 179-185. https://doi.org/ 10.1007/bf02289447.

Hutcheson, G.D., \& Sofroniou, N. (1999). The multivariate social scientist: Introductory statistics using generalized linear models. London: SAGE. https://doi.org/10.1177/096228020000900514.

Kaß, P. (2013). Die Trainertätigkeit im Profifußball - Eine multimethodale Anforderungsanalyse zur Optimierung des Fußball-LehrerLehrgangs (Dissertationsschrift). Köln: Deutsche Sporthochschule

Kauffeld, S. (2006). Kompetenzen messen, bewerten, entwickeln. Stuttgart: Schäffer-Poeschel.

Kauffeld, S., \& Grote, S. (2019). Personalentwicklung. In S. Kauffeld (Hrsg.), Arbeits-, Organisations- und Personalpsychologie für Bachelor (S. 167-210). Berlin, Heidelberg, New York: Springer. https://doi.org/10.1007/978-3-662-56013-6_7.

Kleinbeck, U., \& Kleinbeck, T. (2009). Arbeitsmotivation: Konzepte und Fördermaßnahmen. Lengerich: Pabst Science.

Kline, R. B. (2016). Principles and practice of structural equation modeling (4. Aufl.). Guilford: New York.

Kyndt, E., Govaerts, N., Dochy, F., \& Baert, H. (2011). The learning intention of low-qualified employees: A key for participation in lifelong learning and continuous training. Vocations and Learning, 4(3), 211. https://doi.org/10.1007/s12186-011-9058-5.

Langens, T. A. (2009). Leistung. In V. Brandstätter, J. H. Otto \& J. Bengel (Hrsg.), Handbuch der allgemeinen Psychologie - Motivation und Emotion (S. 217-224). Göttingen: Hogrefe.

Larsen, C.H., Alfermann, D., Henriksen, K., \& Christensen, M.K. (2013). Successful talent development in soccer: The characteristics of the environment. Sport, Exercise, and Performance Psychology, 2(3), 190-206. https://doi.org/10.1037/a0031958.

Lienert, G.A., \& Raatz, U. (1998). Testaufbau und Testanalyse (6. Aufl.). Weinheim: Beltz. 
Livingstone, D. W. (2008). Mapping the field of lifelong (formal and informal) learning and (paid and unpaid) work. In D. W. Livingstone, K. Mirchandani \& P.H. Sawchuk (Hrsg.), The future of lifelong learning and work (S. 13-26). Rotterdam: Brill Sense. https://doi.org/10.1163/9789087904012_004.

MacKenzie, S.B., Podsakoff, P. M., \& Podsakoff, N.P. (2011). Construct measurement and validation procedures in MIS and behavioral research: Integrating new and existing techniques. MIS quarterly, 35(2), 293-334. https://doi.org/10.2307/23044045.

Macnamara, Á., \& Collins, D. (2011). Development and initial validation of the psychological characteristics of developing excellence questionnaire. Journal of Sports Sciences, 29(12), 1273-1286. https://doi.org/10.1080/02640414.2011.589468.

Max, C. (1999). Entwicklung von Kompetenz - ein neues Paradigma für das Lernen in Schule und Arbeitswelt. Frankfurt a.M.: Europäischer Verlag der Wissenschaft.

Mayring, P. (2010). Qualitative Inhaltsanalyse. Grundlagen und Techniken. Weinheim: Beltz. https://doi.org/10.1007/978-3-53192052-8_42.

Mills, A., Butt, J., Maynard, I., \& Harwood, C. (2012). Identifying factors perceived to influence the development of elite youth football academy players. Journal of sports sciences, 30(15), 1593-1604. https://doi.org/10.1080/02640414.2012.710753.

Moosbrugger, H., \& Kelava, A. (2012). Testtheorie und Fragebogenkonstruktion (2. Aufl.). Berlin, Heidelberg, New York: Springer. https://doi.org/10.1007/978-3-642-20072-4.

Moritz, S.E., Feltz, D. L., Fahrbach, K. R., \& Mack, D. E. (2000). The relation of self-efficacy measures to sport performance: A metaanalytic review. Research quarterly for exercise and sport, 71(3), 280-294. https://doi.org/10.1080/02701367.2000.10608908.

Murr, D., Feichtinger, P., Larkin, P., O'Connor, D., \& Höner, O. (2018)a. Psychological talent predictors in youth soccer: A systematic review of the prognostic relevance of psychomotor, perceptual-cognitive and personality-related factors. PloS One, 13(10), 1-24. https://doi.org/10.1371/journal.pone.0205337.

Murr, D., Raabe, J., \& Höner, O. (2018)b. The prognostic value of physiological and physical characteristics in youth soccer: A systematic review. European journal of sport science, 18(1), 62-74. https://doi.org/10.1080/17461391.2017.1386719.

Nesti, M., \& Sulley, C. (2015). Youth development in football: Lessons from the world's best academies. London: Routledge.

R Core Team (2019). R: A language and environment for statistical computing. Wien: R Foundation for Statistical Computing.

Rainbird, H. (2000). Skilling the unskilled: access to work-based learning and the lifelong learning agenda. Journal of education and work, 13(2), 183-197. https://doi.org/10.1080/713676988.

Rigotti, T., Schyns, B., \& Mohr, G. (2008). A short version of the occupational self-efficacy scale: Structural and construct validity across five countries. Journal of Career Assessment, 16(2), 238-255. https://doi.org/10.1177/1069072707305763.

Rosseel, Y. (2012). lavaan. An R package for structural equation modeling. Journal of Statistical Software, 48(2), 1-36. https://doi.org/ 10.18637/jss.v048.i02

Sagar, S. S., Busch, B. K., \& Jowett, S. (2010). Success and failure, fear of failure, and coping responses of adolescent academy football players. Journal of Applied Sport Psychology, 22(2), 213-230. https://doi.org/10.1080/10413201003664962.

Sygusch, R., Liebl, S., \& Töpfer, C. (2014). Die Rahmenrichtlinien für Qualifizierung des Deutschen Olympischen Sportbundes - Hinweise zur Einordnung in den Deutschen Qualifikationsrahmen für lebenslanges Lernen $(D Q R)$. Frankfurt a. M.: Deutscher Olympischer Sportbund.

Van Yperen, N. W. (2009). Why some make it and others do not: Identifying psychological factors that predict career success in professional adult soccer. The Sport Psychologist, 23(3), 317-329. https://doi.org/10.1123/tsp.23.3.317.

Vogel, S., \& Ehemann, T. (2012). Finanzreport deutscher Profisportligen 2012. SPONSORs, 12, 48-59.

Weinert, F.E. (2001). Vergleichende Leistungsmessung in Schulen eine umstrittene Selbstverständlichkeit. In F.E. Weinert (Hrsg.), Leistungsmessungen in Schulen (3. Aufl. S. 17-31). Weinheim: Beltz.

Wendland, M., Elbe, A.-M., Wenhold, F. \& Thonke, F. (2003). AMS Sport: Entwicklung einer Kurzskala (unveröffentlichtes Manuskript). Potsdam: Universität Potsdam

Wenhold, F., Elbe, A.-M., \& Beckmann, J. (2009). Fragebogen zum Leistungsmotiv im Sport (AMS-Sport). Köln: Sportverlag Strauss.

Zuber, C., \& Conzelmann, A. (2014). The impact of the achievement motive on athletic performance in adolescent football players. $\mathrm{Eu}$ ropean Journal of Sport Science, 14(5), 475-483. https://doi.org/ 10.1080/17461391.2013.837513.

Zuber, C., Zibung, M., \& Conzelmann, A. (2015). Motivational patterns as an instrument for predicting success in promising young football players. Journal of Sports Sciences, 33(2), 160-168. https://doi.org/10.1080/02640414.2014.928827.

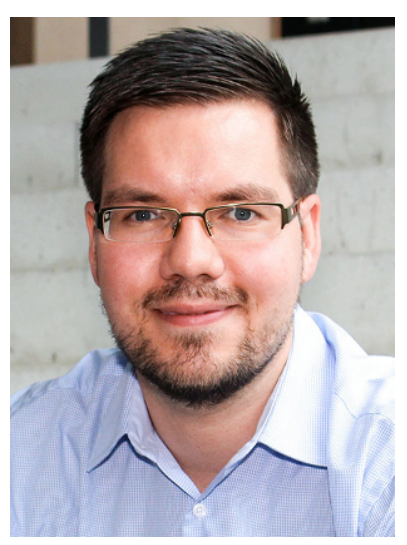

Dr. Julian Decius ist wissenschaftlicher Mitarbeiter am Lehrstuhl für Arbeits- und Organisationspsychologie der Universität Paderborn, wo er zum Thema des informellen Lernens im Kontext industrieller Arbeit promovierte. Er studierte Psychologie mit Vertiefungsrichtung Wirtschaftspsychologie an der Ruhr-Universität Bochum sowie der Lindenwood University, Missouri, USA. Sein Forschungsfokus liegt auf selbstreguliertem und informellem Lernen am Arbeitsplatz und im Studium, strategischer Kompetenzentwicklung in mittelständischen Unternehmen, Weiterbildungsformen unter Berücksichtigung von „Arbeit 4.0“ und „New Work“, sowie Kompetenzerfassung und -förderung bei Profi-Fußballspielern. Praktische Erfahrungen zur Personalentwicklung im Fußball sammelte er u. a. während seiner Tätigkeit in der Bundesliga bei Bayer 04 Leverkusen. 